\title{
RAMIFICATION OF HIGHER LOCAL FIELDS, APPROACHES AND QUESTIONS
}

\author{
LIANG XIAO AND IGOR ZHUKOV
}

This is yet another attempt to organize facts, ideas and problems concerning ramification in finite extensions of complete discrete valuation fields with arbitrary residue fields.

We start $(\S 3)$ with a rather comprehensive description of classical ramification theory describing the behavior of ramification invariants in the case of perfect residue fields. This includes some observations that could be not published earlier, e. g., Prop. 3.3.2 or 3.5.1. We proceed in $\S 4$ with the detailed study of an example showing that almost all the classical theory breaks down if we admit inseparable extensions of residue field and this cannot be easily repaired.

The remaining part of the survey describes several approaches aimed to reproduce parts of the classical theory in the non-classical setting. $\S 5$ is devoted to the description of upper numbering ramification filtration as well as analogs of Artin and Swan conductors in the general case; this description has become standard. Historically, this theory started with the appropriate definitions for abelian extensions via class field theory and cohomological duality and culminated in the general definitions in terms of rigid analytic geometry done by A. Abbes and T. Saito. In $\S 6$, we discuss two ways to realize the ramification filtration geometrically: approach using $l$-adic sheaves as in the work of Abbes and Saito and approach using $p$-adic differential equations as in the work of K. Kedlaya and the first author. Either approach has its own advantage; they are applied to prove important basic theorems on the structure of the ramification filtration. We also introduce the notion of irregularity which is related to one more analogous situation of ramification.

The next section starts with the observation that we still do not have a "fully satisfactory" ramification theory since the upper ramification filtration does not give us enough information about "naïve" invariants including the lower ramification filtration; we sketch some requirements for a "satisfactory theory". We proceed to describe an approach based on the theory of elimination of wild ramification. It results in a construction bearing some properties of classical theory and giving additional information on ramification of given extension. This approach still does not fill the gap but gives some room for further development as mentioned at the end of the section.

Sections 8 and 9 are devoted to the approach of Deligne who started to analyze 2-dimensional ramification problems by looking at all their 1-dimensional restrictions. This makes sense in the context of 2-dimensional schemes, and we suggest to

Date: November 17, 2012.

1991 Mathematics Subject Classification. Primary 11S15; Secondary 14E22.

Key words and phrases. ramification, complete discrete valuation field, two-dimensional local ring, higher local field.

The second author acknowledges financial support from RFBR (project 11-01-00588-a). 
study ramification in an extension of 2-dimensional local fields by "globalizing" the setting, i. e., constructing a sufficiently nice morphism of complete 2-dimensional local rings which serves as a model for given extension. For such morphisms Deligne's idea is applicable: we can look at the induced morphisms of algebroid curves on spectra of 2-dimensional rings and use the classical ramification invariants for them. This study is at the very beginning; there are some initial observations and a lot of open questions.

In Section 10, we discuss the Abbes-Saito upper ramification filtration in the semi-local or global geometric context, for the $l$-adic and $p$-adic realizations as well as for the analogous algebraic $D$-module case. The goal is to compute the Euler characteristic in all three situations in terms of the ramification data, in hope to generalize the Grothendieck-Ogg-Shafarevich formula. Furthermore, we hope to describe or even define log-characteristic cycles using the ramification data.

The last section includes some open questions which we find curious and which are not covered in the previous text.

We almost do not touch here asymptotic properties of ramification numbers in infinite extensions and related notions of deeply ramified or arithmetically profinite extensions except for Subsection 3.10; our subject is restricted to the area of finite extensions of complete fields which still remains full of mystery.

We understand that the subject is not fashionable and in many aspects looks elementary. For this reason, various interesting results, observations, conjectures and questions have good chances to remain unpublished or tend to be forgotten; some of the included questions can already have answers. We would be happy to learn more about what is known and what is unknown; please do not hesitate to send us your comments and suggestions.

We are very grateful to V. Abrashkin, I. Barrientos, D. Benois, I. Faizov, I. Fesenko, E. Lysenko, M. Morrow for valuable remarks.

\section{NOTATION}

If $K$ is a complete discrete valuation field of characteristic 0 or $p$ with the residue field of characteristic $p>0$, the following notation is used.

- $v=v_{K}$ : the valuation on $K$ as well as its (non-normalized) extension to the algebraic closure of $K$;

- $\mathcal{O}_{K}$ : ring of valuation in $K$;

- $\mathfrak{m}_{K}=\left\{a \in \mathcal{O}_{K}: v(a)>0\right\}$ : the maximal ideal of $\mathcal{O}_{K}$;

- $U_{K}=\mathcal{O}_{K}^{*}$

- $U_{i, K}=1+\mathfrak{m}_{K}^{i}, i \geq 1$

- $\pi=\pi_{K}$ : an arbitary uniformizing element of $K$;

- $|\cdot|$ : the norm on $K$ given by $|\pi|^{v(\cdot)}$; when $K$ is of mixed characteristic, we require that $|p|=p^{-1}$;

- $\bar{K}$ : the residue field of $K$;

- $\bar{a}$ : the residue class in $\bar{K}$ of $a \in \mathcal{O}_{K}$;

- $e=e_{K}=v_{K}(p)$ : the absolute ramification index of $K$;

- $K^{\mathrm{ab}}$ : the maximal abelian extension of $K$;

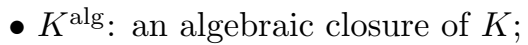

- $G_{K}$ : the absolute Galois group of $K$ (often abbreviated to $G$ when there is no confusion);

- $\zeta_{p^{n}}$ : a primitive $p^{n}$ th root of unity in $K^{\text {alg }}$ (assuming char $K=0$ ). 
For any integral scheme $S, k(S)$ is the field of rational functions on $S$. For an integral domain $A, Q(A)$ is its fraction field.

\section{BASiC DEFINITIONS}

1.1. Ramification invariants. Here we recall various ramification invariants associated with a finite extension $L / K$ where $K$ is a complete discrete valuation field with the residue field $\bar{K}$ of characteristic $p>0$. We shall make a distinction between the classical case when $\bar{K}$ is a perfect (or at least when $\bar{L} / \bar{K}$ is separable) and the non-classical case when this assumption is omitted.

We mention without reference facts proven in $[\mathrm{Se} 68]$ or $[\mathrm{FV}]$; in other cases, proofs or references are usually included.

The most well-known ramification invariants are:

- the ramification index $e(L / K)=v_{L}\left(\pi_{K}\right)$;

- the different $\mathcal{D}_{L / K}$ which can be defined, e. g., as the annihilator ideal of the $\mathcal{O}_{L}$-module of Kähler differentials $\Omega_{\mathcal{O}_{L} / \mathcal{O}_{K}}^{1}$;

- the depth of ramification

$$
d_{M}(L / K)=\inf _{a \in L}\left(v_{M}\left(\operatorname{Tr}_{L / K} a\right)-v_{M}(a)\right),
$$

where $M$ is any finite extension of $K$.

These three invariants are related by the simple formula ([Hy, formula (1-4)]):

$$
v_{L}\left(\mathcal{D}_{L / K}\right)=e(L / K)-1+d_{L}(L / K) .
$$

One of fundamental properties of the depth is its additivity [Hy, Lemma (2-4)]. Namely, for an intermediate field $K^{\prime}$ in $L / K$ we have

$$
d_{M}(L / K)=d_{M}\left(L / K^{\prime}\right)+d_{M}\left(K^{\prime} / K\right) .
$$

We have

$$
[L: K]=e(L / K) f(L / K)=e_{t}(L / K) e_{w}(L / K) f_{s}(L / K) f_{i}(L / K)=e_{t} e_{w} f_{s} f_{i},
$$

where $\left(e_{t}, p\right)=1, e_{w}=p^{N}$ for some $N \geq 0, f_{s}=[\bar{L}: \bar{K}]_{\text {sep }}, f_{i}=[\bar{L}: \bar{K}]_{\text {ins }}$.

A finite extension $L / K$ is said to be:

- unramified, if $[L: K]=f_{s}$;

- totally ramified, if $f_{s}=f_{i}=1$;

- tame, if $e_{w}=f_{i}=1$;

- wild, if $[L: K]=e_{w}$;

- ferocious $^{1}$, if $[L: K]=f_{i}$;

- weakly unramified, if $e_{t}=e_{w}=1$;

- completely ramified, if $e_{t}=f_{s}=1$.

Note that $L / K$ is tame iff $d_{L}(L / K)=0$ [Hy, Remark (2-12)].

If $L / K$ is a Galois extension with the Galois group $G$, for any $g \in G$ one defines the Artin and Swan ramification numbers by the formulas

$$
\begin{aligned}
i(g) & =i_{G}(g)=\inf _{a \in \mathcal{O}_{L}} v_{L}(g(a)-a) ; \\
s(g) & =s_{G}(g)=\inf _{x \in L^{*}} v_{L}\left(g(a) a^{-1}-1\right) .
\end{aligned}
$$

We assume $i_{G}(1)=s_{G}(1)=\infty$.

\footnotetext{
${ }^{1}$ Such extensions are more often referred to as fiercely ramified; this is a translation of original French (Deligne's?) expression "ferocement ramifié". However, John Coates told one of the authors that the English word "ferocious" is more appropriate here than "fierce".
} 
If $\mathcal{O}_{L}$ is generated by $x_{1}, \ldots, x_{n}$ as an $\mathcal{O}_{K}$-algebra, we have

$$
\begin{aligned}
& i_{G}(g)=\inf _{i} v_{L}\left(g\left(x_{i}\right)-x_{i}\right) ; \\
& s_{G}(g)=\inf _{i} v_{L}\left(g\left(x_{i}\right) x_{i}^{-1}-1\right) .
\end{aligned}
$$

In the classical case we have ([Sn], 6.1.4):

$$
s_{G}(g)= \begin{cases}i_{G}(g)-1, & i_{G}(g)>0 \\ 0, & i_{G}(g)=0\end{cases}
$$

On the other hand, if $L / K$ is ferocious, then $s_{G}(g)=i_{G}(g)$ for any $g$.

For an integer $i \geq-1$ the $i$ th ("lower") ramification subgroup is defined as

$$
G_{i}=\left\{\sigma \in G \mid i_{G}(\sigma) \geq i+1\right\} .
$$

More generally, for non-negative integers $n$ and $i$, the $(n, i)$ th ramification subgroup is defined as

$$
G_{n, i}=\left\{\sigma \in G \mid v_{L}(\sigma(x)-x) \geq n+i \text { for all } x \in \mathfrak{m}_{L}^{i}\right\} .
$$

It is a normal subgroup in $G$. It makes sense to consider $G_{n, i}$ with $i>0$ only in the non-classical case. Indeed, in the classical case $G_{n, i}=G_{n-1}$ if $p \mid i$, and $G_{n, i}=G_{n}$ otherwise $([\mathrm{dS}, \S 2])$.

The subgroups $G_{n}=G_{n+1,0}$ and $H_{n}:=G_{n, 1}$ form a filtration on $G$ ([dS, Prop. $2.2-2.3])$ :

$$
G \supseteq G_{0} \supseteq H_{1} \supseteq G_{1} \supseteq H_{2} \supseteq \cdots \supseteq\{1\}
$$

Here $G / G_{0} \simeq \operatorname{Gal}(\bar{L} / \bar{K})$ and $\left(G: G_{0}\right)=f_{s}(L / K) ; G_{0} / H_{1}$ is a cyclic group of order $e_{t}(L / K) ; H_{1}$ is a $p$-group of order $e_{w}(L / K) f_{i}(L / K)$. The subgroups $G_{0}$ and $H_{1}$ will be referred to as inertia subgroup and wild ramification subgroup of $G$ respectively.

For $i>1$, the subgroups $G_{n, i}$ are non-informative, since

$$
G_{n, i}= \begin{cases}H_{n}, & p \nmid i, \\ G_{n-1}, & p \mid i,\end{cases}
$$

when $n>1$, whereas $G_{1, i} / H_{1}$ is exactly the kernel of multiplication by $i$ in the cyclic group $G_{0} / H_{1}$ (see [dS, Prop. 2.3]).

All elements of $\left\{s(\sigma) \mid \sigma \in G_{0}, \sigma \neq 1\right\}$ are said to be ("lower") ramification breaks of $L / K$. If $L / K$ is an inseparable normal extension, the ramification breaks of $L / K$ are defined as $\left\{\right.$ the breaks of $\left.L_{0} / K\right\} \cup\{\infty\}$ where $L_{0} / K$ is the maximal separable subextension of $L / K$.

In the classical case the breaks are exactly the nonnegative integers $i$ with $G_{i} \neq$ $G_{i+1}$. If $\left(G_{i}: G_{i+1}\right)=p^{m}$, then $i$ is called a ramification break of multiplicity $m$.

For a Galois extension $L / K$ in the classical case, the Hasse-Herbrand function $\varphi_{L / K}:[-1, \infty) \rightarrow[-1, \infty)$ is a piecewise linear map defined by the formula

$$
\varphi_{L / K}(u)=\int_{0}^{u} \frac{d t}{\left(G_{0}: G_{t}\right)}
$$

here it is assumed that $G_{i}=G_{[i]+1}$ for non-integral $i$, i. e., in the formula (3) we allow fractional $i$, and $\left(G_{0}: G_{t}\right)=1$ for $t<0$. Since $\varphi_{L / K}$ is strictly increasing, the inverse function $\psi_{L / K}$ is well defined.

It is known that, for a normal subextension $M / K$, we have

$$
\varphi_{L / K}=\varphi_{M / K} \circ \varphi_{L / M}
$$


(It is essential here that we consider the classical case!) Therefore, $\varphi_{L / K}$ can be defined for arbitrary finite separable extension $L / K$ by the formula $\varphi_{L / K}=$ $\varphi_{L^{\prime} / K} \circ \psi_{L^{\prime} / L}$, where $L^{\prime} / K$ is any finite Galois extension containing $L / K$.

Using Hasse-Herbrand function, one defines the "upper" ramification subgroups

$$
G^{u}=G_{\psi_{L / K}(u)} \text { for all } u \geq-1 .
$$

The non-negative rational numbers $u$ such that $G^{v} \neq G^{u}$ for any $v>u$ are called upper ramification breaks of $L / K$. The biggest such $u$ is called the highest ramification break, denoted by $b(L / K)$.

The upper ramification breaks are exactly the ordinates of points on the graph of $\varphi_{L / K}$ where the slope is changed, whereas the lower ramification breaks are their abscissas. The number 0 is a break if and only if $e_{t} \neq 1$; the other breaks are called wild. A change of slope by factor $p^{m}$ corresponds to a wild break of multiplicity $m$. This property can be used as a definition of lower and upper breaks for non-Galois finite extensions $L / K$. (In this case even the lower breaks need not be integral.)

1.1.1. Example. Let $L / K$ be a totally ramified cyclic extension of degree $p^{n}$, and let $s_{1}<\cdots<s_{n}$ be all Swan ramification numbers of $L / K$. Then $L / K$ have $n$ upper breaks $h_{1}<\cdots<h_{n}$, all of multiplicity 1 , and

$$
h_{r}=s_{1}+\sum_{i=2}^{r} \frac{s_{i}-s_{i-1}}{p^{i-1}}=\sum_{i=1}^{r} \frac{p-1}{p^{i}} s_{i}+\frac{1}{p^{r}} s_{r} .
$$

1.2. $m$-dimensional complete discrete valuation fields. We give only definitions; see [HLF, Ch. I] for more information.

Let $K$ be a field. We define a structure of an $m$-dimensional complete discrete valuation field ( $m$-CDVF) on $K$ as a sequence of fields $k_{m}=K, k_{m-1}, \ldots, k_{0}$ such that $k_{i}$ is a complete discrete field with the residue field $k_{i-1}, 1 \leq i \leq m$. The field $k_{m-1}\left(\right.$ resp. $\left.k_{0}\right)$ is referred to as the first (resp. the last) residue field of $K$.

If the last residue field is perfect, $K$ is said to be an $m$-dimensional local field. (NB: often it is required that the last residue field is finite.)

A system of local parameters of $K$ is any $m$-tuple $t_{1}, \ldots, t_{m}$ such that each $t_{i}$ is a lifting to $K$ of some uniformizing element of $k_{i}$.

Fix a system of local parameters $t_{1}, \ldots, t_{m}$ and consider the map

$$
\mathbf{v}_{K}=\left(v_{1}, \ldots, v_{m}\right): K^{*} \rightarrow \mathbb{Z}^{m}
$$

where $v_{m}=v_{k_{m}}, v_{m-1}(\alpha)=v_{k_{m-1}}\left(\alpha_{m-1}\right), \alpha_{m-1}$ is the class of $\alpha t_{m}^{-v_{m}(\alpha)}$ in $k_{m-1}$, etc. Then $\mathbf{v}_{K}$ is a discrete valuation of rank $m$; here it is assumed that $\mathbb{Z}^{m}$ is lexicographically ordered as follows: $\mathbf{i}=\left(i_{1}, \ldots, i_{m}\right)<\mathbf{j}=\left(j_{1}, \ldots, j_{m}\right)$, if and only if

$$
i_{l}<j_{l}, i_{l+1}=j_{l+1}, \ldots, i_{m}=j_{m} \text { for some } l \leq m \text {. }
$$

If we change the system of local parameters, the valuation is replaced by an equivalent one. Thus, $\mathbf{v}_{K}$ is defined up to equivalence.

For any finite extension $L / K$, there exists a unique structure of an $m$-dimensional complete discrete valuation field on $L$ compatible with that on $K$; the non-normalized $\left(\mathbb{Q}^{m}\right.$-valued) extension of $\mathbf{v}_{K}$ on $L$ is also denoted by $\mathbf{v}_{K}$.

The notion of depth of ramification can be generalized as follows ([Hy, (1-3)]):

$$
\mathbf{d}_{M}(L / K)=\inf _{a \in L}\left(\mathbf{v}_{M}\left(\operatorname{Tr}_{L / K} a\right)-\mathbf{v}_{M}(a)\right),
$$

where both $L$ and $M$ are finite extensions of $K$. 


\section{CyClic extensions of Degree $p$ And Genome}

2.1. Cyclic extensions of degree $p$. Here we look carefully at the case of a Galois extension $L / K$ with $[L: K]=p$ (see also [Hy, Lemma (2-16)]). This is important for discussing examples in the subsequent sections.

Let $g$ be any generator of $G=\operatorname{Gal}(L / K)$; then $i(g)$ and $s(g)$ are independent of the choice of $g$, and we can use the notation $s(L / K)=s(g)$.

Since $[L: K]=e_{t} e_{w} f_{s} f_{i}$, and $e_{t}$ is prime to $p$, there can be 3 cases.

Case U (unramified): $f_{s}=p, e_{w}=f_{i}=1$. Here $i(g)=s(g)=0$.

Case W (wild): $e_{w}=p, f_{s}=f_{i}=1$. Let $s=v_{L}\left(\pi_{L}^{g} / \pi-1\right)$. Then $\mathcal{O}_{L}=\mathcal{O}_{K}[\pi]$ immediately implies $i(g)=s+1, s(g)=s$.

Case F (ferocious): $f_{i}=p, f_{s}=e_{w}=1$. Choose any $t \in \mathcal{O}_{L}$ such that $\bar{t} \notin \bar{K}$. Let $s=v_{L}\left(t^{g} / t-1\right)$. Then $\mathcal{O}_{L}=\mathcal{O}_{K}[t]$ and $i(g)=s(g)=s$.

In all 3 cases we have $d_{L}(L / K)=(p-1) s(L / K)$.

Let us compute ramification invariants for specific constructions of cyclic extension of degree $p$, i. e., for Artin-Schreier and Kummer extensions.

$1^{\circ}$. $\operatorname{char} K=p$. In this case $L=K(x), \wp(x)=a \in K$, where $\wp=X^{p}-X$. We have $v(a) \leq 0$ since $\mathfrak{m}_{K} \subset \wp(K)$ by Hensel lemma. Choose an equation with maximal possible $v(a)$.

If $v(a)=0$, the Hensel lemma implies $\bar{a} \notin \wp(\bar{K})$, and we are in the Case U.

If $v(a)<0$ and $p \nmid v(a)$, we obviously have Case $\mathrm{W}$, and $s(L / K)=-v(a)$.

If $v(a)<0$ and $p \mid v(a)$, the maximality of $v(a)$ implies $\overline{\pi^{-v(a)} a} \notin \bar{K}^{p}$. It follows that we have Case $\mathrm{F}$, and $s(L / K)=-v(a) / p$.

$2^{\circ}$. char $K=0, \zeta_{p} \in K$. In this case $L=K(x), x^{p}=a$. We can choose $a$ with $v(a)=1$ or $v(a)=0$; in the latter case we require that $l=v(a-1)$ is maximal. Then we can distinguish 5 cases.

A. $v(a)=1$. Here we have Case $\mathrm{W}$,

$$
s(L / K)=v_{L}\left(\zeta_{p}-1\right)=\frac{e_{L}}{p-1}=\frac{p e}{p-1} .
$$

B. $v(a)=0, \bar{a} \notin \bar{K}^{p}$. This is Case $\mathrm{F}$,

$$
s(L / K)=v_{L}\left(\zeta_{p}-1\right)=\frac{e_{L}}{p-1}=\frac{e}{p-1} .
$$

C. $v(a)=0, \bar{a}=1, l<\frac{p e}{p-1}, p \nmid l$. This is Case $\mathrm{W}, s(L / K)=\frac{p e}{p-1}-l$.

D. $v(a)=0, \bar{a}=1, l<\frac{p e}{p-1}, p \mid l$. From the maximality of $l$ it follows that this is Case $\mathrm{F}, s(L / K)=\frac{1}{p}\left(\frac{p e}{p-1}-l\right)$.

E. $v(a)=0, \bar{a}=1, l \geq \frac{p e}{p-1}$. It follows from Hensel lemma that in fact $l=\frac{p e}{p-1}$, and this is Case $\mathrm{U}$.

2.2. Genome of an extension. Let $L / K$ be a cyclic extension of degree $p^{n}$. Then it can be uniquely written as a tower $L=M_{n} / M_{n-1} / \ldots / M_{1} / M_{0}=K$ of cyclic extensions of degree $p$, and the genome of $L / K$ is by definition the word $T_{1} \ldots T_{n}$, where

$$
T_{i}= \begin{cases}W, & M_{i} / M_{i-1} \text { wild } \\ F, & M_{i} / M_{i-1} \text { ferocious. }\end{cases}
$$

We would be able define the genome for a general Galois extension of degree $p^{n}$, were a positive answer to the following question known. 
2.2.1. Question. Let $L / K$ be a completely ramified Galois extension, $G=\operatorname{Gal}(L / K)$; $G_{i}$ and $H_{i}$ the ramification subgroups of $G$ defined in previous section. Denote by $K_{i}$ (resp. $L_{i}$ ) the intermediate field fixed by $H_{i}$ (resp. $G_{i}$ ). Is it true that any $L_{i} / K_{i}$ is ferocious and any $K_{i+1} / L_{i}$ is wild?

\section{WhAT IS NICE IN THE CLASSICAL CASE}

Throughout this section we consider only the case of perfect $\bar{K}$. We list various facts which are sometimes referred to as "beautiful ramification theory" in the classical case. (However, probably the whole collection of facts has not been ever included in one text.)

3.1. Factor groups. Let $K^{\prime}$ be an intermediate field in $L / K$. Then the ramification invariants of $K^{\prime} / K$ can be described in terms of those of $L / K$. More specifically, let $L / K$ be a finite Galois extension with $G=\operatorname{Gal}(L / K)$, and $K^{\prime}$ an intermediate extension corresponding to a normal subgroup $H$. Then for any $\sigma \in G / H, \sigma \neq 1$, we have

$$
i_{G / H}(\sigma)=\frac{1}{e_{L / K^{\prime}}} \sum_{s H=\sigma} i_{G}(s)
$$

(Herbrand theorem, see [Se68, Ch. IV, Prop. 3]).

It follows that we have the following statement comparing lower and upper ramification filtrations on $G / H$ with those on $G$.

3.1.1. Proposition. 1. For any $v \geq-1$ we have $(G / H)_{v}=G_{\psi_{L / K^{\prime}}(v)} H / H$.

2. For any $v \geq-1$ we have $(G / H)^{v}=G^{v} H / H$.

3.1.2. Corollary. Let $H=G_{j}$ for some $j$. Then

$$
(G / H)_{i}= \begin{cases}G_{i} / H, & i \leq j \\ \{1\}, & i \geq j\end{cases}
$$

One of the nice consequences of Prop. 3.1.1 is that we can define upper ramification filtration for an infinite Galois extensions $L / K$ by the formula

$$
\operatorname{Gal}(L / K)^{v}=\lim _{\substack{L^{\prime} / \overleftarrow{K} \text { finite } \\ L^{\prime} \subset L}} \operatorname{Gal}\left(L^{\prime} / K\right)^{v}
$$

In particular, we have an upper ramification filtration on the whole absolute Galois group.

3.2. Subgroups. Let $L / K$ be a finite Galois extension, and $K^{\prime} / K$ any subextension; $G=\operatorname{Gal}(L / K), H=\operatorname{Gal}\left(L / K^{\prime}\right)$. Obviously, $H_{i}=G_{i} \cap H$ for any $i$. Therefore,

$$
H^{i}=H_{\psi_{L / K^{\prime}}(i)}=G_{\psi_{L / K^{\prime}}(i)} \cap H=G^{\varphi_{L / K} \circ \psi_{L / K^{\prime}}(i)} \cap H=G^{\varphi_{K^{\prime} / K}(i)} \cap H .
$$

3.3. Base change. Here we observe how the ramification invariants change as one passes from $L / K$ to $L K^{\prime} / K^{\prime}$ for some finite extension $K^{\prime} / K$ linearly disjoint with $L / K$. We start with the basic case of two Galois extensions of degree $p$. 
3.3.1. Lemma. 1. Let $L_{1} / K$ and $L_{2} / K$ be Galois extensions of degree $p$ with positive $s_{1}=s\left(L_{1} / K\right)$ and $s_{2}=s\left(L_{2} / K\right)$, and $s_{1}<s_{2}$. Then $s\left(L_{1} L_{2} / L_{2}\right)=s_{1}$, and $s\left(L_{1} L_{2} / L_{1}\right)=s_{1}+p\left(s_{2}-s_{1}\right)$.

2. Let $L_{1} / K$ and $L_{2} / K$ be linearly disjoint Galois extensions of degree $p$ such that $s=s(L / K)>0$ is the same for any subextension $L / K$ of degree $p$ in $L_{1} L_{2} / K$. Then $s\left(L_{1} L_{2} / L_{2}\right)=s\left(L_{1} L_{2} / L_{1}\right)=s$.

Proof. Set $L=L_{1} L_{2}, G=\operatorname{Gal}(L / K)$.

Assume first that $L / K$ has two distinct lower ramification breaks $s_{1}^{\prime}<s_{2}^{\prime}$. Put $H_{2}=G_{s_{1}^{\prime}+1}, K^{\prime}=L^{H_{2}}$. Then by Cor. 3.1.2 we have

$$
\operatorname{Gal}\left(K^{\prime} / K\right)_{i}= \begin{cases}\operatorname{Gal}\left(K^{\prime} / K\right), & i \leq s_{1}^{\prime}, \\ \{1\}, & i>s_{1}^{\prime}\end{cases}
$$

whence $s\left(K^{\prime} / K\right)=s_{1}^{\prime}$.

Let $K^{\prime \prime} / K$ be any other subextension of degree $p$ in $L / K, H=\operatorname{Gal}\left(L / K^{\prime \prime}\right), \sigma_{0}$ any element of $G$ outside $H$. Note that $\sigma_{0} H$ contains an only element of $H_{2}$, with Artin number $s_{2}^{\prime}+1$. By (5),

$$
i_{G / H}\left(\left.\sigma_{0}\right|_{K^{\prime \prime}}\right)=\frac{1}{p}\left((p-1) \cdot\left(s_{1}^{\prime}+1\right)+1 \cdot\left(s_{2}^{\prime}+1\right)\right)=s_{1}^{\prime}+\frac{s_{2}^{\prime}-s_{1}^{\prime}}{p}+1 .
$$

It follows $s\left(K^{\prime \prime} / K\right)=s_{1}^{\prime}+\frac{s_{2}^{\prime}-s_{1}^{\prime}}{p}$. Since $s_{1}$ and $s_{2}$ are among $s\left(K^{\prime} / K\right)$ and (all) $s\left(K^{\prime \prime} / K\right)$, and $s_{1}<s_{2}$, we conclude that $s_{1}=s_{1}^{\prime}, s_{2}=s_{1}^{\prime}+\frac{s_{2}^{\prime}-s_{1}^{\prime}}{p}$.

In the remaining case when $L / K$ has one break $s^{\prime}$ of multiplicity 2 , the same computation shows that $s\left(K^{\prime \prime} / K\right)=s^{\prime}$ for any subextension $K^{\prime \prime} / K$ of degree $p$ in $L / K$.

This can be generalized as follows.

3.3.2. Proposition. Let $L / K$ and $K^{\prime} / K$ be finite Galois p-extensions. Assume that $L / K$ have upper ramification breaks $h_{1}, \ldots, h_{r}$ with multiplicities $m_{1}, \ldots, m_{r}$. Assume that all the upper ramification breaks of $K^{\prime} / K$ are distinct from $h_{1}, \ldots, h_{r}$. Then the upper ramification breaks of $L K^{\prime} / K^{\prime}$ are $\psi_{K^{\prime} / K}\left(h_{1}\right), \ldots, \psi_{K^{\prime} / K}\left(h_{r}\right)$ and their multiplicities are $m_{1}, \ldots, m_{r}$.

Proof. For $[L: K]=\left[K^{\prime}: K\right]=p$, this is the first part of Lemma 3.3.1. The general case follows by double induction on $[L: K]$ and $\left[K^{\prime}: K\right]$.

3.3.3. Question. If $L / K$ and $K^{\prime} / K$ are Galois extensions of degree $p$ with the same ramification break, we cannot determine the ramification invariants of $L K^{\prime} / K^{\prime}$ in general. However, in view of the second part of Lemma 3.3.1, we can do this if we know the ramification breaks of all subextensions of degree $p$ in $L K^{\prime} / K$.

How can this observation be generalized to arbitrary finite Galois $p$-extensions $L / K$ and $K^{\prime} / K$ ?

3.4. Filtration on the group of units and the norm map. For a finite extension $L / K$, consider the norm map $N_{L / K}: L^{*} \rightarrow K^{*}$ and its interaction with the filtration on $K^{*}$ by the groups $U_{i, K}, i \geq 1$, and the similar filtration on $L^{*}$. For any $i \geq 1$, define $f(i)$ by the conditions

$$
N_{L / K} U_{i, L} \subset U_{f(i), K}, \quad N_{L / K} U_{i, L} \not \subset U_{f(i)+1, K} .
$$


Then the map $f=f_{L / K}$ can be computed from the ramification breaks of $L / K$ and vice versa, at least if the residue field $\bar{K}$ is infinite. Indeed, [FV, Prop. (3.1)] states essentially the following.

3.4.1. Proposition. Let $\bar{K}$ be infinite, $L / K$ a finite Galois extension, and $\psi=$ $\psi_{L / K}$. Then for any positive integer $j$ we have $f(i)=j$, if $\psi(j-1)+1 \leq i \leq \psi(j)$.

3.4.2. Remark. Thus, for infinite $\bar{K}, f_{L / K}(i)$ is equal to the minimal integer not less than $\varphi_{L / K}(i)$. If $\bar{K}$ is finite, $f_{L / K}(i)$ can "jump" at the lower ramification breaks of $L / K$.

3.4.3. Question. How can $\varphi_{L / K}$ be defined in terms of $f_{L / K}$ in the case of finite $\bar{K} ?$

3.4.4. Question. What is the exact relation between $f_{L / K}$ and $\varphi_{L / K}$ for a nonGalois $L / K$ ?

3.5. Artin-Schreier and Kummer filtrations and the embedding map. First assume char $K=p$. Then we have a filtration on $K / \wp(K)$ by the groups

$$
C_{i, K}=\left(\mathfrak{m}_{K}^{i}+\wp(K)\right) / \wp(K), \quad i \leq 0 .
$$

(Recall that $\mathfrak{m}_{K} \subset \wp(K)$ by Hensel lemma.) Then, for a finite extension $L / K$, we can consider the interaction of this filtration with a similar one on $L / \wp(L)$. For $i \geq 0$, determine $g(i)$ by the conditions $\varepsilon\left(C_{-i, K}\right) \subset C_{-g(i), L}, \varepsilon\left(C_{-i, K}\right) \not \subset C_{-g(i)+1, L}$ for the natural map $\varepsilon: K / \mathfrak{p}(K) \rightarrow L / \mathfrak{p}(L)$.

In the same spirit, if char $K=0, \zeta_{p} \in K$, we can consider filtration on $K^{*} /\left(K^{*}\right)^{p}$ by the subgroups

$$
C_{i, K}^{*}=U_{i, K}\left(K^{*}\right)^{p} /\left(K^{*}\right)^{p}, \quad 1 \leq i \leq \frac{p e}{p-1} .
$$

(Recall that $U_{\frac{p e}{p-1}+1, K} \subset\left(K^{*}\right)^{p}$.) For a finite $L / K$ and a positive integer $i<$ $\frac{p e}{p-1}$, define $g(i)$ by the conditions $\varepsilon\left(C_{\frac{p e}{p-1}-i, K}^{*}\right) \subset C_{\frac{p e}{p-1}-g(i), L}^{*}$ and $\varepsilon\left(C_{\frac{p e}{p-1}-i, K}^{*}\right) \not \subset$ $C_{\frac{p e}{p-1}-g(i)+1, L}^{*}$ for the natural map $\varepsilon: K^{*} /\left(K^{*}\right)^{p} \rightarrow L^{*} /\left(L^{*}\right)^{p}$.

Then the function $g=g_{L / K}$ is closely related to $\psi=\psi_{L / K}$. Namely, Prop. 3.3.2 and explicit computation of the ramification break for an Artin-Schreier or Kummer extension immediately imply

3.5.1. Proposition. Let $i$ be a positive integer which is prime to $p$ and distinct from any upper ramification break of $L / K$. (We also require $i<\frac{p e}{p-1}$ if char $\left.K=0.\right)$ Then $g(i)=\psi(i)$.

If $\bar{K}$ is infinite, we can use the second part of Lemma 3.3.1 to prove

3.5.2. Proposition. For an infinite $\bar{K}$, let $i$ be a positive integer which is prime to p. $\left(i<\frac{p e}{p-1}\right.$ if char $K=0$.) Then $g(i)=\psi(i)$.

Since the upper breaks are always prime to $p$, this means that $g$ determines the ramification invariants of $L / K$ in the case of infinite $\bar{K}$ and char $K=p$.

Similarly, if $\zeta_{p^{n}} \in K$, one can define an explicit filtration on $K^{*} /\left(K^{*}\right)^{p^{n}}$ compatible with the upper ramification filtration on the maximal abelian extension of $K$ of exponent $p$. 
3.5.3. Question. Can we construct $\psi_{L / K}$ from the filtrations on $K\left(\zeta_{p^{n}}\right)^{*} /\left(K\left(\zeta_{p^{n}}\right)^{*}\right)^{p^{n}}$ for all $n$, thus eliminating the condition $i<\frac{p e}{p-1}$ in Prop. 3.5.2?

If char $K=p$, the explicit form of the filtration on $W_{r}(K) / \wp\left(W_{r}(K)\right)$ compatible with the ramification filtration is given in $[\mathrm{Br}, \S 1]$. Here $W_{r}$ denotes the group Witt vectors of length $r$, and

$$
\wp\left(\left(x_{0}, \ldots, x_{r-1}\right)\right)=\left(x_{0}^{p}, \ldots, x_{r-1}^{p}\right)-W_{r}(K)\left(x_{0}, \ldots, x_{r-1}\right) ;
$$

note that Jean-Luc Brylinski uses different notation. For a new proof and very clear treatment of related questions, see [Th].

\subsection{Hasse-Arf theorem.}

3.6.1. Theorem. Let $L / K$ be a finite abelian extension. Then all upper ramification breaks of $L / K$ are integral.

See [Se68, Ch. IV, §3], [FV, Ch. III, (4.3)].

An inverse result is due to Fesenko [Fe95b]:

3.6.2. Proposition. Let $L / K$ be a totally ramified finite Galois extension such that for any totally ramified finite abelian extension $K^{\prime} / K$ all upper ramification breaks of $L K^{\prime} / K^{\prime}$ are integral. Then $L / K$ is abelian.

3.6.3. Question. Can we replace the class of all abelian extensions $K^{\prime} / K$ by a smaller class here, e. g., by the class of all elementary abelian extensions, at least in the case $\operatorname{char} K=p$ ?

3.6.4. Question. For a finite Galois extension $L / K$, can we determine $\operatorname{Gal}(L / K)$, if we know all upper ramification breaks of $L K^{\prime} / K^{\prime}$ for all abelian extensions $K^{\prime} / K$ ?

One of the related results is the following Sen congruence (see, e. g., [Sn, Theorem 6.1.34]).

3.6.5. Proposition. Let $L / K$ be a finite Galois extension, $g \in \operatorname{Gal}(L / K)$ such that $s(g)>0$ and $g^{p^{n}} \neq 1$. Then

$$
s\left(g^{p^{n-1}}\right) \equiv s\left(g^{p^{n}}\right) \quad \bmod p^{n} .
$$

3.7. Artin and Swan representations. (See [Se68, Ch. VI], [Se77] as well as the discussion in [Sn, 6.1].) Fix a finite Galois extension $L / K$, and for $G=\operatorname{Gal}(L / K)$ introduce Artin and Swan central function $a_{G}, s w_{G}: G \rightarrow \mathbb{Z}$ by formulas

$$
\begin{aligned}
a_{G}(\sigma) & = \begin{cases}-f \cdot i_{G}(\sigma), & \sigma \neq 1, \\
f \sum_{\tau \neq 1} i_{G}(\tau), & \sigma=1,\end{cases} \\
s w_{G}(\sigma) & = \begin{cases}-f \cdot s_{G}(\sigma), & \sigma \neq 1, \\
f \sum_{\tau \neq 1} s_{G}(\tau), & \sigma=1,\end{cases}
\end{aligned}
$$

where $f=f(L / K)$.

The Serre's theorem on the existence of Artin representation ([Se77, p. 68]) claims:

3.7.1. Proposition. The central functions $a_{G}$ and sw $w_{G}$ are characters of certain complex representations of $G$. 
For the corresponding representations $A_{G}$ and $S W_{G}$ we have the following explicit formulas in the ring of complex representations $R(G)$ (cited from [Sn, 6.1]):

$$
A_{G}=\sum_{i=0}^{\infty}\left[G_{0}: G_{i}\right]^{-1} \operatorname{Ind}_{G_{i}}^{G}\left(\operatorname{Ind}_{\{e\}}^{G}(1)-1\right)
$$

and

$$
S W_{G}=A_{G}+\operatorname{Ind}_{G_{0}}^{G}(1)-\operatorname{Ind}_{\{e\}}^{G}(1)
$$

where $\operatorname{Ind}_{H}^{G}(V)$ denotes the representation of $G$ induced by the representation $V$ of $H$, and 1 is the class of 1 -dimensional trivial representation of the corresponding group.

For a normal subgroup $H$ of $G$ it follows from Herbrand theorem that

$$
S W_{G / H} \simeq S W_{G} \otimes_{\mathbb{C}[G]} \mathbb{C}[G / H] .
$$

For the character $\chi$ of a complex representation $V$ of $G$, the Artin conductor of $\chi($ or $V)$ is defined as

$$
\operatorname{Ar}_{K}(\chi)=\operatorname{Ar}_{K}(V)=\left\langle a_{G}, \chi\right\rangle_{G}=\frac{1}{|G|} \sum_{g \in G} a_{G}(g) \overline{\chi(g)} .
$$

Similarly, the Swan conductor of $\chi($ or $V$ ) is

$$
\operatorname{Sw}_{K}(\chi)=\operatorname{Sw}_{K}(V)=\left\langle s w_{G}, \chi\right\rangle_{G}=\frac{1}{|G|} \sum_{g \in G} s w_{G}(g) \overline{\chi(g)} ;
$$

we have

$$
\operatorname{Sw}_{K}(V)=\operatorname{Ar}_{K}(V)+\operatorname{dim} V^{G_{0}}-\operatorname{dim} V .
$$

3.7.2. Example. (see [Se68, Ch. VI, Prop. 5]) Let $L / K$ be a totally ramified cyclic extension of degree $p^{n}$, and $\chi$ the character of any faithful (i. e., injective) representation of $G=\operatorname{Gal}(L / K)=\langle g\rangle$. Let $s_{1}<\cdots<s_{n}$ be all Swan ramification numbers of $L / K$. Then

$$
\begin{aligned}
\mathrm{Sw}_{K}(\chi) & =\frac{1}{p^{n}} \sum_{i=1}^{p^{n}} \zeta^{i} s w_{G}\left(g^{i}\right) \\
& =\frac{1}{p^{n}} \sum_{r=0}^{n} \sum_{v_{p}(i)=r} \zeta^{i} s w_{G}\left(g^{i}\right) \\
& =-\frac{1}{p^{n}} \sum_{r=0}^{n-1} s_{r+1} \sum_{v_{p}(i)=j} \zeta^{i}+\frac{1}{p^{n}} \sum_{i=1}^{p^{n}-1} s_{G}\left(g^{i}\right) \\
& =\frac{1}{p^{n}}\left(s_{n}+\sum_{r=0}^{n-1}\left(p^{n-r}-p^{n-r-1}\right) s_{r+1}\right) \\
& =b(L / K)
\end{aligned}
$$

in view of (4), where $\zeta$ is a primitive $p^{n}$ th root of unity in $\mathbb{C}$.

3.7.3. Remark. This is the simplest case of the following fact (see [Se68, Ch. VI, $\S 2$, Ex. 2]). Let $V$ be an irreducible representation of $G$ of dimension $d$. Then $\operatorname{Ar}_{K}(V)=d(b(L / K)+1)$, where $b(L / K)$ is the highest (upper) ramification break defined in Section 1.1. 
As a consequence of this fact, we may define the Artin conductor and Swan conductor of a finite dimensional complex representation $V$ of $G$ to be

$$
\operatorname{Ar}_{K}(V)=\sum_{a \geq-1}(a+1) \cdot \operatorname{dim} V^{G^{a+}} / V^{G^{a}}, \quad \operatorname{Sw}_{K}(V)=\sum_{a \geq 0} a \cdot \operatorname{dim} V^{G^{a+}} / V^{G^{a}} .
$$

Note that one can recover the ramification filtration on $G$ from Artin conductors of all its irreducible representations. (The same does not hold for Swan conductors since Swan conductor measures only wild ramification and does not know anything about $\left(G_{0}: G_{1}\right)$.)

In a similar way, one defines Swan conductor for $\mathbb{F}_{l}$-representations; this version of Swan conductor is used in Grothendieck-Ogg-Shafarevich formula (see Subsection 3.11 below).

There is an alternative and equivalent way of stating Proposition 3.7.1.

3.7.4. Proposition. For all finite dimensional complex representation $V$ of $G$, the Artin conductor $\operatorname{Ar}_{K}(V)$ and the Swan conductors $\operatorname{Sw}_{K}(V)$ are non-negative integers.

Applying this to all one-dimensional representations of $G$ and using the above explicit description of Artin and Swan conductors (Remark 3.7.3), we obtain that $b(L / K)$ is always an integer for an abelian extension $L / K$. Thus, we recover the original Hasse-Arf Theorem 3.6.1. So sometimes the above proposition will be also referred to as Hasse-Arf theorem.

3.8. Local class field theory. Let $K$ be a complete discrete valuation field of any characteristic with a quasi-finite residue field of prime characteristic. (A field $F$ is called quasi-finite if $G_{F} \simeq \widehat{\mathbb{Z}}$.)

The central theorem of local class field theory states that there exists a homomorphism $\Theta_{K}: K^{*} \rightarrow \operatorname{Gal}\left(K^{\mathrm{ab}} / K\right)$ uniquely determined by the following two properties.

1. For any finite abelian extension $L / K, \Theta_{K}$ induces an isomorphism $\Theta_{L / K}$ : $K^{*} / N_{L / K} L^{*} \rightarrow \operatorname{Gal}(L / K)$.

2. For any prime element $\pi_{K}$, the restriction of $\Theta_{K}\left(\pi_{K}\right)$ on the maximal unramified extension of $K$ is the Frobenius automorphism.

It appears that the reciprocity map transforms the valuation filtration on the multiplicative group into the upper ramification filtration on (abelian) Galois group. More precisely, we have the following results. ([Se68], Ch. XV, Th.1 with Cor. 3 and Th. 2. Note that $N_{L / K} U_{\psi(n), L} \subset U_{n, K}$ by Prop. 3.4.1.)

3.8.1. Proposition. Let $L / K$ be a finite abelian extension. Put $\psi=\psi_{L / K}$.

1. For any positive integer $n$, the canonical map $U_{n, K} / N_{L / K} U_{\psi(n), L} \rightarrow K^{*} / N_{L / K} L^{*}$ is injective.

2. The reciprocity map $\Theta_{L / K}$ transforms the filtration on $K^{*} / N_{L / K} L^{*}$ by subgroups $U_{n, K} / N_{L / K} U_{\psi(n), L}$ into the filtration on $G=\operatorname{Gal}(L / K)$ by $G^{n}$.

3.8.2. Proposition. Let $L / K$ be a possibly infinite abelian extension, $G=\operatorname{Gal}(L / K)$. For any positive integer $n$ the image of $\Theta_{K}\left(U_{n, K}\right) \subset \operatorname{Gal}\left(K^{\mathrm{ab}} / K\right)$ in $G$ is dense in $G^{n}$ (equal to $G^{n}$ if the residue field of $K$ is finite).

In characteristic 0 , provided $\zeta_{p} \in K$, this implies self-duality of the valuation filtration on $K^{*} /\left(K^{*}\right)^{p}$ with respect to Hilbert symbols. In characteristic $p$, we 
have a duality between the valuation filtration on $K^{*} /\left(K^{*}\right)^{p^{r}}$ and the Brylinski filtration on $W_{r}(K) / \wp\left(W_{r}(K)\right)$, see [Br, Theorem 1].

For Fesenko's non-abelian reciprocity map [Fe01], compatibility with ramification filtration was established in [IS].

3.9. Abrashkin's anabelian yoga. Let $K_{1}$ and $K_{2}$ be local fields (complete discrete valuation fields with finite residue fields). V. A. Abrashkin [Abr00], [Abr10] proved that if there exists an isomorphism between absolute Galois groups of $K_{1}$ and $K_{2}$ preserving ramification filtration, then $K_{1}$ and $K_{2}$ are isomorphic. (Characteristic 0 case is due to Sh. Mochizuki [Mo-S].)

3.10. A theorem of Deligne. Let $K$ and $K^{\prime}$ be two complete discrete valuation fields (typically with large absolute ramification indices in the case of mixed characteristic). Assume that there exists $b \in \mathbb{N}$ such that there is an isomorphism $\mathcal{O}_{K} / \pi_{K}^{b} \mathcal{O}_{K} \cong \mathcal{O}_{K^{\prime}} / \pi_{K^{\prime}}^{b} \mathcal{O}_{K^{\prime}}$ as rings. Deligne [De84] proved the following result.

3.10.1. Proposition. Keep the notation as above. If $\bar{K}$ has a perfect residue field, then there is a canonical isomorphism

$$
G_{K} / G_{K}^{b} \cong G_{K^{\prime}} / G_{K^{\prime}}^{b}
$$

In other words, the quotient Galois groups above depend only on the truncated discrete valuation rings $\mathcal{O}_{K} / \pi_{K}^{b} \mathcal{O}_{K} \cong \mathcal{O}_{K^{\prime}} / \pi_{K^{\prime}}^{b} \mathcal{O}_{K^{\prime}}$. Note that there were no assumptions on the characteristics of $K$ and $K^{\prime}$. In particular, they could be different, which may be used to build a connection between the mixed characteristic fields and the equal characteristic fields on the aspect of ramification theory.

Deligne's theorem provides an alternative way to understand the field of norms of J.-M. Fontaine and J.P. Wintenberger [FW1, FW2] (which precedes Deligne's work)

Put $K_{n}=\mathbb{Q}_{p}\left(\zeta_{p^{n}}\right)$ for $n \in \mathbb{N}$ and $K_{\infty}=\cup_{n \in \mathbb{N}} K_{n}$. We take the uniformizer $\pi_{K_{n}}$ to be $\zeta_{p^{n}}-1$. Then the tower $\left(K_{n}\right)_{n \in \mathbb{N}}$ is APF (short for arithmetically profinite) in the sense of [FW1, FW2]. The following statement is a special case of the main result of Fontaine-Wintenberger [FW1, FW2] (exposed also in [FV, Ch. III, Theorem 5.7]).

3.10.2. Theorem. There is a canonical isomorphism between the absolute Galois group of $K_{\infty}$ and that of the equal characteristic field $\mathbb{F}_{p}((T))$.

One can give a heuristic proof using Deligne's theorem as follows. For each $n$, we put $r_{n}=p^{n-1}(p-1)$ so that $\mathcal{O}_{K_{n}} / \pi_{K_{n}}^{r_{n}} \cong \mathbb{F}_{p} \llbracket T \rrbracket /\left(T^{r_{n}}\right)$. Deligne's theorem then implies that we have an isomorphism

$$
G_{\mathbb{F}_{p}((T))} / G_{\mathbb{F}_{p}((T))}^{r_{n}} \cong G_{K_{n}} / G_{K_{n}}^{r_{n}} .
$$

An easy computation shows that $\varphi_{K_{n} / K}(n)=r_{n}$. The basic property in Subsection 3.2 implies that $G_{K_{n}}^{r_{n}}=G_{\mathbb{Q}_{p}}^{n} \cap G_{K_{n}}$. Thus, taking the inverse limit of (8) gives an isomorphism between $G_{\mathbb{F}_{p}((T))}$ and $G_{K_{\infty}}$.

We expect that the same proof works for general complete discrete valuation field $K$ in place of $\mathbb{Q}_{p}$, at least when $K$ has a perfect residue field, and hence we could reprove the main result of [FW1, FW2] this way. The APF condition is expected to ensure that the inverse limit of (8) as $n \rightarrow \infty$ gives the isomorphism between the Galois group of $K_{\infty}$ and that of $\bar{K}((T))$. Unfortunately, to our knowledge, we do not know if such a proof exists in the literature. 
3.11. Global formulas. Let $\mathcal{X}$ be a smooth projective curve over an algebraically closed field and let $\mathcal{Y}$ be its normalization in a finite extension of $k(\mathcal{X})$. RiemannHurwitz formula compares the genera of these curves:

$$
2 g_{\mathcal{Y}}-2=[k(\mathcal{Y}): k(\mathcal{X})]\left(2 g_{\mathcal{X}}-2\right)+\sum_{Q} v_{Q}\left(\mathcal{D}_{\mathcal{Y} / \mathcal{X}}\right)
$$

where $Q$ runs over closed points of $\mathcal{Y}$.

Let $U$ be a dense open subset of $\mathcal{X}, \bar{\eta}$ a geometric generic point of $\mathcal{X}, \mathcal{F}$ a locally constant sheaf of $\mathbb{F}_{l}$-modules of finite rank on $U_{\text {ét }}$. Then the geometric generic fiber $M=\mathcal{F}_{\bar{\eta}}$ is a finite-dimensional $\mathbb{F}_{l}$-representation of $\operatorname{Gal}(k(\mathcal{X}))$; it factors through $\operatorname{Gal}(L / k(\mathcal{X}))$, where $L / k(\mathcal{X})$ is a finite Galois extension.

For a closed point $P$ of $\mathcal{X}$, the Swan conductor $\mathrm{Sw}_{P} \mathcal{F}$ is defined as the Swan conductor of $M$ considered as $\operatorname{Gal}\left(L_{w} / k(\mathcal{X})_{v}\right)$-module, where $v$ corresponds to $P$, and $w$ is any extension of $v$ to $L$. Independence of $L$ follows from an $\mathbb{F}_{l}$-analog of (6). Then the Grothendieck-Ogg-Shafarevich formula for $\mathcal{F}$ reads:

$$
\chi_{c}(U, \mathcal{F})=\chi_{c}\left(U, \mathbb{F}_{l}\right) \operatorname{rank} \mathcal{F}-\sum_{P \in \mathcal{X} \backslash U} \operatorname{Sw}_{P} \mathcal{F},
$$

where $\chi_{c}(U, \cdot)$ is the Euler-characteristic of the corresponding étale sheaf. (This can be obtained from the shape of G.-O.-S. formula in [Mil] as follows. Let $u: U \hookrightarrow \mathcal{X}$, $\mathcal{F}_{0}$ a constant sheaf on $U_{\text {ét }}$ of rank equal to rank $\mathcal{F}$. Apply the formula in [Mil, Ch. V, Th. 2.12] to both $u ! \mathcal{F}$ and $u ! \mathcal{F}_{0}$ and compute the difference.)

For equivariant versions of Riemann-Hurwitz and Grothendieck-Ogg-Shafarevich formulas, see [Kö].

3.11.1. Remark. We point out that there is an analogous statement for lisse $\mathbb{Q}_{l^{-}}$ sheaves instead of lisse $\mathbb{F}_{l}$-sheaves. ${ }^{2}$ In fact the formula for the former reduces to that of the latter, as we explain now.

A lisse $\mathbb{Q}_{l}$-sheaf $\mathcal{F}$ corresponds to a representation $\rho: \pi_{1}(U) \rightarrow \mathrm{GL}_{d}\left(\mathbb{Q}_{l}\right)$. Since the fundamental group is profinite and hence compact, the image $\rho\left(\pi_{1}(U)\right)$ lands in $\mathrm{GL}_{d}\left(\mathbb{Z}_{l}\right)$ (up to conjugation). This integral representation $\rho^{\circ}$ gives rise to a lisse $\mathbb{Z}_{l}$-sheaf $\mathcal{F}^{\circ}$. Put $\bar{\rho}=\rho^{\circ} \bmod l$ and $\overline{\mathcal{F}}=\mathcal{F}^{\circ} / l$. It is not difficult to show that the Euler characteristic of $\mathcal{F}$ agrees with that of $\overline{\mathcal{F}}$. We need to match the Swan conductors.

Note that, for each point $P \in \mathcal{X} \backslash U$, the wild ramification group $W_{P}$ at $P$ is a pro$p$ group; but the kernel of $\mathrm{GL}_{d}\left(\mathbb{Z}_{l}\right) \rightarrow \mathrm{GL}_{d}\left(\mathbb{F}_{l}\right)$ is a pro-l group. Hence the image $\rho\left(W_{P}\right)$ has trivial intersection with $\operatorname{Ker}\left(\mathrm{GL}_{d}\left(\mathbb{Z}_{l}\right) \rightarrow \mathrm{GL}_{d}\left(\mathbb{F}_{l}\right)\right)$; consequently, we have an isomorphism $\rho\left(W_{P}\right) \cong \bar{\rho}\left(W_{P}\right)$. From this it is clear that $\mathrm{Sw}_{P} \mathcal{F}=\mathrm{Sw}_{P} \overline{\mathcal{F}}$, since both sides depend only on the action of the wild inertia group.

3.12. Completeness. Given a finite Galois extension of complete discrete valuation fields $L / K$ with $\operatorname{Gal}(L / K)=G$, we have a number of ramification invariants occurring in various formulas: $e(L / K), v_{L}\left(\mathcal{D}_{L / K}\right), G_{i}$ and $G^{i}$ for $i \geq 0, \operatorname{Ar}_{K}(V)$ and $\operatorname{Sw}_{K}(V)$ for a complex representation $V$ of $G$. However, there is a sufficient system of ramification invariants, namely, the lower ramification filtration, which "describes the ramification completely": all the other ramification invariants (including local terms of classical global formulas) can be expressed in terms of it.

\footnotetext{
${ }^{2}$ We can of course consider a finite extension of $\mathbb{Q}_{l}$ in place of $\mathbb{Q}_{l}$; the argument goes through with no essential changes.
} 
(Upper ramification filtration is a sufficient system of invariants as well. The same is true for Artin conductors of all complex representations of $G$.) For example,

$$
\begin{gathered}
e(L / K)=\left|G_{0}\right| ; \\
v_{L}\left(\mathcal{D}_{L / K}\right)=\sum_{i=0}^{\infty}\left|G_{i}\right|-1 ;
\end{gathered}
$$

and

$$
\operatorname{Sw}_{K}(V)=\sum_{i=1}^{\infty} \frac{1}{\left(G: G_{i}\right)} \operatorname{dim}_{\mathbb{C}}\left(V / V^{G_{i}}\right),
$$

where $V$ is a finite-dimensional complex representation of $G$.

\section{WHAT IS MISSING IN THE NON-CLASSICAL CASE}

This section is devoted to the detailed study of an example of extension $L / K$ with $\operatorname{Gal}(L / K) \simeq(\mathbb{Z} / p)^{2}$ such that for $L / K$ Lemma 3.3.1 (as well as any reasonable analog of it) fails. Furthermore, the example exhibits obstacles to extension of the most part of classical theory to the general case.

Let us look carefully at the following example.

Let $K$ be a complete discrete valuation field of characteristic $p>0$ with imperfect residue field. Fix a prime element $\pi$ and $t \in \mathcal{O}_{K}$ such that $\bar{t} \notin \bar{K}^{p}$. Take some positive integers $N>n>m$ such that $N \equiv n \equiv-1(\bmod p)$. Now we define $L_{1} / K$ and $L_{2} / K$ by Artin-Schreier equations:

$$
\begin{array}{ll}
K_{1}=K\left(x_{1}\right), & x_{1}^{p}-x_{1}=a_{1}=\pi^{-n}+\pi^{-m} t, \\
K_{2}=K\left(x_{2}\right), & x_{2}^{p}-x_{2}=\pi^{-N},
\end{array}
$$

and set $L=K_{1} K_{2}=K\left(x_{1}, x_{2}\right)=K_{1}\left(x_{2}\right)=K_{2}\left(x_{1}\right)$.

In view of considerations in Section 2 , both $K_{1} / K$ and $K_{2} / K$ are wild, and $s\left(K_{1} / K\right)=n, s\left(K_{2} / K\right)=N$. Note also that for any subextension $K^{\prime} / K$ of degree $p$ in $L / K$ we have $s\left(K^{\prime} / K\right)=N$ unless $K^{\prime}=K_{1}$.

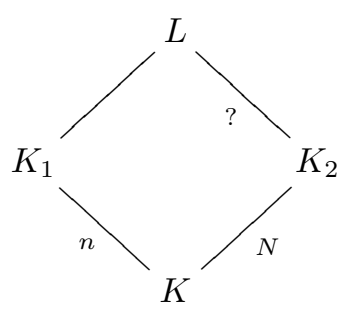

Let us compute $s\left(L / K_{2}\right)$. Put $N=p D-1$. Then $\pi_{2}=x_{2} \pi^{D}$ is a prime in $K_{2}$, and the equation

$$
\left(\pi^{D} x_{2}\right)^{p}-\pi^{(p-1) D}\left(\pi^{D} x_{2}\right)=\pi
$$

implies

$$
\pi=\pi_{2}^{p}-\pi_{2}^{(p-1) p D+1}+\cdots,
$$


where the dots denote terms of higher order. Thus,

$$
\begin{aligned}
a_{1} & =\left(\pi_{2}^{p}-\pi_{2}^{(p-1) p D+1}+\cdots\right)^{-n}+\left(\pi_{2}^{p}-\pi_{2}^{(p-1) p D+1}+\cdots\right)^{-m} t \\
& =\pi_{2}^{-p n}\left(1-\pi_{2}^{(p-1) p D-p+1}+\cdots\right)^{-n}+\pi_{2}^{-p m}\left(1-\pi_{2}^{(p-1) p D-p+1}+\cdots\right)^{-m} t \\
& =\pi_{2}^{-p n}\left(1+n \pi_{2}^{(p-1) N}+\cdots\right)+\pi_{2}^{-p m}\left(1+m \pi_{2}^{(p-1) N}+\cdots\right) t \\
& =\pi_{2}^{-p n}+n \pi_{2}^{-p n+(p-1) N}+\cdots+\pi_{2}^{-p m} t+\cdots \\
& \equiv \underbrace{\pi_{2}^{-n}}_{-n}+n \underbrace{\pi_{2}^{-p n+(p-1) N}}_{-p n+(p-1) N}+\cdots+\underbrace{\pi_{2}^{-p m} t}_{-p m}+\cdots\left(\bmod \wp\left(K_{2}\right)\right),
\end{aligned}
$$

where the numbers under the braces denote the corresponding values of $v_{K_{2}}$.

Assume further that $m>\frac{n}{p}$. Since $-n<-p n+N(p-1)$, the valuation of the sum is $-p m$. We can conclude that $L / K_{2}$ is ferocious, and $s\left(L / K_{2}\right)=m$. Note that the latter number is not determined by the values of $n=s\left(K_{1} / K\right)$ and $N=s\left(K_{2} / K\right)$. (However, if $m<\frac{n}{p}$, the valuation of the sum is $-n$, the extension $L / K_{2}$ is wild and $s\left(L / K_{2}\right)=m$. In fact, we are in the classical case here.)

We see that an analog of Lemma 3.3.1 is not true in the general case: we cannot predict $s\left(L / K_{2}\right)$ even having known the $s\left(K^{\prime} / K\right)$ for any subextension $K^{\prime} / K$ of degree $p$ in $L / K$.

Next, the "compatibility with factor groups" property also fails in the general case. Indeed, from the depth additivity (2) we have

$$
d_{L}(L / K)=d_{L}\left(L / K_{2}\right)+d_{L}\left(K_{2} / K\right)=(p-1) m+(p-1) N,
$$

and

$$
d_{L}\left(L / K_{1}\right)=d_{L}(L / K)-d_{L}\left(K_{1} / K\right)=(p-1)(m+N)-(p-1) n,
$$

whence $s\left(L / K_{1}\right)=m+N-n$. Therefore, the two breaks of the (lower) ramification filtration of $L / K$ are $m$ and $m+N-n$, and these two numbers do not give enough information to determine, say, $s\left(K_{1} / K\right)=n$.

Essentially, this example shows that we cannot give a suitable definition of "upper ramification filtration" based on the usual (Artin or Swan) ramification numbers, and consequently we lose all constructions and facts using this upper filtration: Hasse-Arf theorem, Artin and Swan representations, global formulas etc.

Also, we do not have any "completeness" for the known systems of invariants. In particular, one of the motivating goals in development of "non-classical" ramification theory could be to obtain an explicit form for the order of different (or, equivalently, for the depth of ramification) in terms of suitable lower or upper ramification breaks, i. e., an analog of (10).

For more examples showing "mysterious behavior" of ramification invariants in the non-classical case, see [Hy], [Sn, 6.2], [Lo].

\section{Kato-Swan CONDUCtor AND its Generalizations}

5.1. Kato-Swan conductor. Note that for abelian extensions of usual local fields the upper filtration can be recovered from the filtration on the multiplicative group by Prop. 3.8.1. In the same way one could define an upper filtration in the situations where some class field theory is available, e. g., for abelian extensions of higher local fields with finite last residue field (see [HLF] for the basic facts about higher 
local fields and [Fe96] for a survey of various versions of higher local class field theory). This approach was explored in several papers starting from [Lo]. For example, Hyodo [Hy] defines ("upper") ramification breaks for a finite abelian extension $L / K$ of $m$-dimensional local fields (with finite last residue field) as $m$-tuples

$$
\mathbf{j}_{L / K}(l)= \begin{cases}\max \left\{\mathbf{i} \in \mathbb{Z}_{+}^{m}:\left|\Theta_{L / K}\left(U_{\mathbf{i}} K_{m}^{\text {top }} K\right)\right| \geq p^{l}\right\}, & \text { if such } \mathbf{i} \text { exists }, \\ 0, & \text { otherwise, }\end{cases}
$$

for all $l \geq 1$, where $\Theta_{L / K}: K_{m}^{\text {top }} K \rightarrow \operatorname{Gal}(L / K)$ is the reciprocity map and $\left(U_{\mathbf{i}} K_{m}^{\text {top }} K\right)$ is the standard filtration on $K_{m}^{\text {top }} K$ defined by means of valuation of rank $m$. For the case of arbitrary perfect last residue field, see [Fe95a, §4].

In a compatible manner with this observation, Kato [Ka89] introduced a notion of a conductor for one-dimensional representations of $\operatorname{Gal}(L / K)$, where $L / K$ is a finite extension of a complete discrete valuation field with any residue field.

We do not include Kato's definition, since it is difficult to do this in a selfcontained manner; see, e. g., [Sn, 6.2]. However, his conductor $\operatorname{KSw}(\chi)$ can be characterized by either of the following two properties ([Sp99], Prop. 3.3.10 and Cor. 3.3.11).

5.1.1. Proposition. Let $\chi \in H^{1}(K)$ be a character of $G=\operatorname{Gal}\left(K^{\mathrm{ab}} / K\right)$; denote by $L_{\chi}$ the subfield in $K^{\mathrm{ab}}$ fixed by $\chi$.

1. $\mathrm{KSw}_{G}(\chi)$ is the smallest integer $n \geq 0$ such that $\left\{\chi_{L_{0}}, u\right\}=0$ in $\operatorname{Br} L_{0}$ for any $u \in U_{n+1, L_{0}}$, where $L_{0}$ is the maximal unramified subextension in $L_{\chi} / K$.

2. $\mathrm{KSw}_{G}(\chi)$ is the smallest integer $n \geq 0$ such that $U_{n+1, K} \subset N_{L_{\chi} / K} L_{\chi}^{*}$.

Here $H^{1}(K)=\operatorname{Hom}\left(\operatorname{Gal}\left(K^{\mathrm{ab}} / K\right), \mathbb{Q} / \mathbb{Z}\right)$; the braces denote the cohomological pairing $H^{1}(K) \times K^{*} \rightarrow H^{2}(K)=\operatorname{Br} K$.

From this, one can define a filtration $G^{\bullet}$ on $G=\operatorname{Gal}\left(K^{\mathrm{ab}} / K\right)$ so that, for any character $\chi$ of $G$, we have

$$
\operatorname{KSw}_{G}(\chi)=\inf \left\{a>0 \mid G^{a} \subseteq \operatorname{Ker} \chi\right\}
$$

we call this filtration the Kato filtration on $G$.

For an $m$-dimensional local field $K$ with finite last residue field and $\chi \in H^{1}(K)$, $\mathrm{KSw}_{G}(\chi)$ is exactly the smallest integer $n \geq 0$ such that $\Theta_{L / K}\left(U_{n+1} K_{m}^{\text {top }} K\right)$ acts trivially on $L_{\chi}$, see $[\operatorname{Sp} 99,3.4]$. In other words, $\operatorname{KSw}_{G}(\chi)$ is the last component of the maximal break $\mathbf{j}(1)$ for $L_{\chi} / K$ in Hyodo's notation (12).

In the classical case this Kato-Swan conductor coincides with the usual Swan conductor. This relation between KSw and the usual (Swan) ramification numbers is in force also in the so called Case II (cf. Subsection 7.2), [Ka89, prop. 6.8, p.12]:

5.1.2. Proposition. Let $L / K$ be a finite Galois extension, $G=\operatorname{Gal}(L / K)$, and $\chi: G \rightarrow \mathbb{C}^{*}$ a one-dimensional representation. Assume that either $\bar{L} / \bar{K}$ is separable or $e(L / K)=1$ and $\bar{L} / \bar{K}$ is generated by one element. Then

$$
\operatorname{KSw}_{G}(\chi)=-\frac{1}{e(L / K)} \sum_{g \in G} s(g) \chi(g),
$$

where we use the convention that $s(1)=-\sum_{g \in G, g \neq 1} s(g)$.

Boltje, Cram and Snaith (see [BCS], [Sn, 6.3]) define a conductor in the general case by means of explicit Brauer induction. This results in a conductor compatible with Swan conductor and Kato-Swan conductor in the cases where those are defined. 
5.2. Borger's construction using generic perfection. J. Borger [Bo04, Bo02] constructed a conductor through considering a "generic residual perfection" process. His result is based on the following observation: taking $\mathcal{O}_{K}=\mathbb{F}_{p}(x) \llbracket \pi \rrbracket$ as an example, one would like to reduce the definition of ramification filtrations to the case of perfect residue field, by adjoining $p^{\infty}$-roots of $x$. Note that $x$ should be thought of as a lift of the $x$ of the residue field. But there is no canonical such lift, as one could choose, for example, $x+\pi$ instead and adjoin all $p^{\infty}$-roots of $x+\pi$. Borger's idea is to introduce an indeterminate $u_{1}$ and consider $\mathbb{F}_{p}\left(x, u_{1}\right) \llbracket y \rrbracket$; he then adjoins all $p^{\infty}$-roots of $x+u_{1} \pi$. Next, he has to deal with $p$-power roots of $u_{1}$. For this, he adjoins another indeterminate $u_{2}$ and all $p^{\infty}$-roots of $u_{1}+u_{2} \pi$. Continuing this process and "taking limit" gives a "generic perfection of $\mathcal{O}_{K}$ ".

To present this observation systematically, Borger showed that there is a moduli space $\operatorname{Spf}\left(A^{\mathrm{u}}\right)$ that parametrizes the ways of modifying $\mathcal{O}_{K}$ so that its residue field is perfect. In the example above,

$$
A^{\mathrm{u}}=\mathbb{F}_{p}(x)\left[u_{1}, u_{2}, \ldots\right]\left[\left(x+u_{1} \pi\right)^{1 / p^{\infty}},\left(u_{1}+u_{2} \pi\right)^{1 / p^{\infty}}, \ldots\right] \llbracket \pi \rrbracket .
$$

Let $A^{\mathrm{g}}$ denote the the completion of $A^{\mathrm{u}}$ at the generic point of its special fiber. Then $Q\left(A^{\mathrm{g}}\right)$ is a complete discrete valuation field with perfect residue field.

We may then use the natural map $G_{K} \rightarrow G_{Q\left(A^{g}\right)}$ to pull back the (upper) ramification filtration on the latter group. Borger [Bo04] proved that this construction is compatible with the "non-logarithmic" (Artin-like) version of Kato conductor.

5.3. Approach of A. Abbes and T. Saito. A. Abbes and T. Saito [AS02] adopted a rather different approach to the construction of (upper) ramification filtration using rigid analytic spaces. (We refer $[\mathrm{BGR}]$ for basics of rigid analytic spaces.)

As explained in Section 4, it seems very difficult to obtain the upper ramification breaks from the lower ones when the residue field $\bar{K}$ is not perfect. Abbes and Saito, instead, work directly with the upper ramification filtrations. Their construction relies on the following crucial but easy proposition in the case of perfect residue field case.

5.3.1. Proposition. Let $K$ be a complete discrete valuation field with perfect residue field. Let $L$ be a finite Galois extension of $K$ with Galois group $G=G(L / K)$. We know that $\mathcal{O}_{L}$ is generated as an $\mathcal{O}_{K}$-algebra by one element $x$. Let $P(u)$ be the minimal polynomial of $x$.

(i) Let $b(L / K)$ be the highest ramification break as defined just before Example 1.1.1. We assume that $L / K$ is not unramified so that $b(L / K) \geq 0$. Then

$$
b(L / K)=\frac{1}{e(L / K)}\left(\sum_{g \in G, g \neq 1} v_{L}(g(x)-x)+\max _{g \in G, g \neq 1} v_{L}(g(x)-x)\right) .
$$

(ii) Consider the rigid analytic space for each positive rational number a:

$$
X^{a}=\left\{\left.u \in K^{\mathrm{alg}}|| u|\leq 1,| P(u)|\leq| \pi_{K}\right|^{a}\right\} .
$$

Then $X^{a}$ has $[L: K]$ geometric connected components if and only if $a>b(L / K)$.

Proof. The first statement is straightforward from unwinding the definition of upper ramification filtration.

A rigorous proof of (ii) can be found in [AS02, Lemma 6.6]. We will give a rough idea of why this is true. The picture here is that, if $a$ is very large, we confine $u$ 
in very small neighborhoods of the roots of $P(u)=0$, which are the conjugates of $x$. The rigid space $X^{a}$ is expected to be geometrically a disjoint union of very small discs centered at each of conjugates of $x$. In other words, $X^{a}$ should have $[L: K]$ geometric connected components. In contrast, when $a \rightarrow 0^{+}$, the condition $|P(u)|<\left|\pi_{K}\right|^{a}$ is significantly weakened, and $X^{a}$ is almost the whole disc $|u| \leq 1$.

When the rational number $a$ decreases from a big starting value, the discs grow larger. There is a first such a moment that some of the $[L: K]$ discs clash together, and the number of geometric connected components decreases. We need to show that the rational number $a$ at this moment is exactly the highest ramification break $b(L / K)$. Indeed, the cut-off condition is obviously $|u-x|<\min _{g \in G, g \neq 1}|g x-x|$ (or with a conjugate of $x$ in place of $x$ ). This implies that $|u-g x|=|g x-x|$ for $g \neq 1$. Thus

$$
|P(u)|=\left|\prod_{g \in G}(u-g x)\right|=\prod_{g \in G}|u-g x|=|u-x| \prod_{g \in G}|g x-x|<\left|\pi_{K}\right|^{b(L / K)} .
$$

In fact, one can turn this explanation into a complete proof if argued more carefully.

Trying to imitate this description in the general case, Abbes and Saito considered the following construction. Let $K$ be a complete discrete valuation field and $L$ a finite Galois extension of $K$. Suppose that $\mathcal{O}_{L}$ is generated by $x_{1}, \ldots, x_{r}$ as an $\mathcal{O}_{K^{-}}$ algebra. Then we may write $\mathcal{O}_{L}$ as the quotient $\mathcal{O}_{K}\left[u_{1}, \ldots, u_{r}\right] /\left(f_{1}, \ldots, f_{s}\right) \simeq \mathcal{O}_{L}$, where the isomorphism sends $u_{i}$ to $x_{i}$. For a positive rational number $a$, consider the following rigid analytic space

$$
X_{L / K}^{a}:=\left\{\underline{u}=\left(u_{1}, \ldots, u_{r}\right) \in\left(K^{\mathrm{alg}}\right)^{r} \mid \begin{array}{c}
\left|u_{1}\right| \leq 1, \ldots,\left|u_{r}\right| \leq 1 ; \\
\left|f_{1}(\underline{u})\right| \leq\left|\pi_{K}\right|^{a}, \ldots,\left|f_{s}(\underline{u})\right| \leq\left|\pi_{K}\right|^{a}
\end{array}\right\} .
$$

Put $G=G_{K}$ for simplicity. Inspired by Prop. 5.3.1, we want to define an (upper) ramification filtration $G^{b}$ of $G$ so that $X_{L / K}^{a}$ has $[L: K]$ geometric connected components if and only if $a>\inf \left\{b \mid G^{b} \subseteq G_{L}\right\}$. It is not difficult to see that the space $X_{L / K}^{a}$ does not depend on the choice of $f_{i}$ 's, and the set of geometric connected components $\pi_{0}^{\text {geom }}\left(X_{L / K}^{a}\right)$ does not depend on the choice of $u_{i}$ 's (because adding a new generator is equivalent to changing $X_{L / K}^{a}$ to a fiber bundle over $X_{L / K}^{a}$ with discs as the fibers.) So our statement is well-defined, depending only on $L$.

Abbes and Saito [AS02] proved the existence of such ramification filtration using certain abstract framework of "Galois functor" by studying functors for all rational $a$ that take every finite Galois extension $L$ of $K$ to the set of geometric connected components $\pi_{0}^{\text {geom }}\left(X_{L / K}^{a}\right)$; they call it the non-logarithmic ramification filtration $G_{\text {nlog }}^{a}$ for $a \in \mathbb{Q}_{\geq 0}$. They also gave a log-variant of the construction which gives the logarithmic ramification filtration $G_{\log }^{a}$ for $a \in \mathbb{Q}_{\geq 0}$. For details, we refer to [AS02].

We list a few immediate properties of these filtrations using notation $G_{\text {nlog }}^{a+}$ (resp. $G_{\log }^{a+}$ ) for the closure of union of $G_{\text {nlog }}^{b}\left(\right.$ resp. $\left.G_{\log }^{b}\right)$ over all $b>a$ :

(1) both filtrations are left continuous, with rational breaks;

(2) for $0<a \leq 1, G_{\text {nlog }}^{a}$ is the inertia subgroup of $G$ (inverse limit of inertia subgroups over finite subextensions);

(3) $G_{\mathrm{nlog}}^{1+}=G_{\log }^{0+}$ is the wild ramification subgroup of $G$ (inverse limit of wild ramification subgroups over finite subextensions); 
(4) if $\bar{K}$ is perfect, we have $G_{\mathrm{nlog}}^{a+1}=G_{\log }^{a}=G^{a}$ for all $a \geq 0$; here $\left(G^{a}\right)$ is usual (upper) ramification filtration;

(5) if $K^{\prime} / K$ is a finite unramified extension, then both filtrations on $G_{K^{\prime}}$ are induced by those on $G_{K}$;

(6) if $K^{\prime} / K$ is a finite tame extension with $e\left(K^{\prime} / K\right)=m$, then $\left(G_{K^{\prime}}\right)_{\log }^{m a}=$ $\left(G_{K}\right)_{\log }^{a}$ for any $a$;

(7) if $K^{\prime} / K$ is any finite extension with $e\left(K^{\prime} / K\right)=m$, then $\left(G_{K^{\prime}}\right)_{\log }^{m a} \subset\left(G_{K}\right)_{\log }^{a}$ for any $a$.

For $L / K$ a finite Galois extension, we put $b_{\text {nlog }}(L / K)=\inf \left\{b \mid G_{\text {nlog }}^{b} \subseteq G_{L}\right\}$ and $b_{\log }(L / K)=\inf \left\{b \mid G_{\log }^{b} \subseteq G_{L}\right\}$; they are called the highest non-log and log (upper) ramification break, respectively. The following is a typical example of ramification breaks.

5.3.2. Example. Let $K=\bar{K}((\pi))$ be an equal characteristic complete discrete valuation field and let $L=K(z)$ be an Artin-Schreier extension given by $z^{p}-z=$ $a \pi^{-n}$ for $a \in \bar{K} \llbracket \pi \rrbracket^{*}$ and $n \in \mathbb{N}$. We assume that the generator $z$ is chosen so that $n$ is minimal (see $\S 2$ ). The Galois group $\operatorname{Gal}(L / K) \cong \mathbb{Z} / p \mathbb{Z}$.

(1) If $p \nmid n$, then we have $b_{\text {nlog }}(L / K)=n+1$ and $b_{\log }(L / K)=n$.

(2) If $p \mid n$, then we have $b_{\log }(L / K)=b_{\operatorname{nlog}}(L / K)=n$.

B. Chiarellotto and A. Pulita $[\mathrm{ChP}]$ proved that the induced filtration $G_{\log }^{\mathrm{ab}, \bullet}$ on the abelian Galois group $G^{\text {ab }}$ agrees with the Kato filtration in Subsection 5.1.

For a finite dimensional representation $\rho: G_{K} \rightarrow \mathrm{GL}(V)$ with finite image, we put $b_{\text {nlog }}(\rho)=b_{\text {nlog }}(V):=b_{\text {nlog }}(L / K)$ and $b_{\log }(\rho)=b_{\log }(V):=b_{\log }(L / K)$, where $L$ is the finite extension of $K$ corresponding to the kernel of $\rho$. Under very mild technical restrictions, these ramification filtrations also enjoy the following HasseArf property, as proved in [X10, X12a].

5.3.3. Theorem. Assume either $K$ is of equal characteristic, or $p>2$ and $K$ is not absolutely unramified (i.e. $p$ is not an uniformizer). Let $\rho: G_{K} \rightarrow \operatorname{GL}(V)$ be an irreducible representation with finite image. Then the Swan conductor $\operatorname{Sw}(\rho):=$ $b_{\log }(\rho) \cdot \operatorname{dim} \rho$ and the Artin conductor $\operatorname{Art}(\rho):=b_{\operatorname{nlog}}(\rho) \cdot \operatorname{dim} \rho$ are integers.

We will come back to the discussion of this proof later in Subsection 6.2.

In the same paper [X10], it is also proved that, when $K$ is of equal characteristic, Abbes and Saito nonlog filtration agrees with Borger filtration. In the mixed characteristic case, a similar argument used in [X10] relates Abbes and Saito non-log filtration with a variant of Borger filtration (see [X12a, Remark 3.2.14]). It would be interesting to see if the two definitions are exactly the same.

As of yet, we are not aware of any attempt to compare the approach of BoltjeCram-Snaith with the other two.

We also mention that Abbes-Saito's construction can be applied to finite flat group schemes over $\mathcal{O}_{K}$ and give a ramification filtration on the group schemes. For progress along this line, see [AM, Ha12, Ha12+]. This result can be used to prove the existence of canonical subgroups for a $p$-divisible group with small degree; see $[\mathrm{Ti}]$.

5.4. Refined Swan conductors. A much less trivial result says that the factors of these filtrations $\operatorname{gr}^{a} G_{\mathrm{nlog}}^{\bullet}:=G_{\mathrm{nlog}}^{a} / G_{\mathrm{nlog}}^{a+}(a>1)$ and $\operatorname{gr}^{a} G_{\log }^{\bullet}:=G_{\log }^{a} / G_{\log }^{a+}(a>0)$ are abelian groups of exponent $p$. This was proved with some restrictions in [AS03], 
[Sa09], [X12a] and, in full generality, in [Sa12]. Moreover, there is a natural injective homomorphism

$$
\text { rsw : } \operatorname{Hom}\left(\operatorname{gr}^{a} G_{\log }^{\bullet}, \mathbb{F}_{p}\right) \hookrightarrow \Omega_{\mathcal{O}_{K}}^{1}(\log ) \otimes_{\mathcal{O}_{K}} \mathfrak{m}_{\bar{K}}^{-a} / \mathfrak{m}_{\bar{K}}^{(-a)+}, \quad a \in \mathbb{Q}_{>0},
$$

where $\Omega_{\mathcal{O}_{K}}^{1}(\log ):=\Omega_{\mathcal{O}_{K}}^{1}+\mathcal{O}_{K} \frac{d \pi_{K}}{\pi_{K}}, \mathfrak{m}_{\bar{K}}^{-a}:=\left\{x \in K^{\text {alg }} \mid v_{K}(x) \geq-a\right\}$, and $\mathfrak{m}_{\bar{K}}^{(-a)+}:=\left\{x \in K^{\mathrm{alg}} \mid v_{K}(x)>-a\right\}$. Following Kato, this map is called the refined Swan conductor homomorphism. When $K$ is of equal characteristic, one can define an analogous refined Artin conductor homomorphism

$$
\operatorname{rar}: \operatorname{Hom}\left(\operatorname{gr}^{a} G_{\mathrm{nlog}}^{\bullet}, \mathbb{F}_{p}\right) \hookrightarrow \Omega_{\mathcal{O}_{K}}^{1} \otimes_{\mathcal{O}_{K}} \mathfrak{m}_{\bar{K}}^{\frac{-a}{K}} / \mathfrak{m}_{\bar{K}}^{(-a)+}, \quad a \in \mathbb{Q}_{>1} .
$$

See $[\mathrm{X} 12 \mathrm{~b}]$ for more details. The analogous refined Artin conductor homomorphism is also expected in the mixed characteristic case, using a variant of argument of [Sa12].

When $\bar{K}$ is finite and $a$ an integer, the rsw map is compatible with the natural homomorphism in local class field theory in the following way.

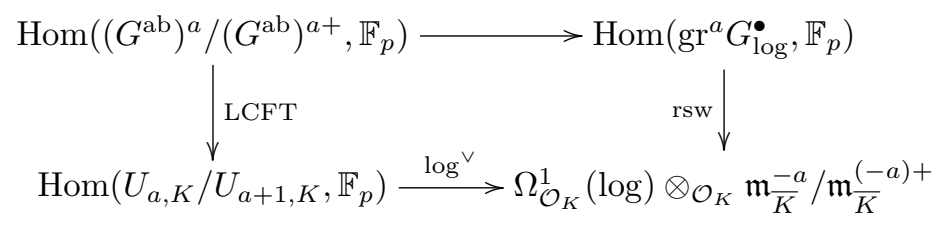

where $G^{\text {ab }}$ denotes the abelian Galois group with the induced filtration, the left vertical map is the isomorphism from the local class field theory, and the map $\log \vee$ is characterized below. For a homomorphism $\eta: U_{a, K} / U_{a+1, K} \rightarrow \mathbb{F}_{p}$, its image $\log ^{\vee}(\eta)$ is the element $w_{\eta} \pi_{K}^{-a} \frac{d \pi_{K}}{\pi_{K}}$ for $w_{\eta} \in \bar{K}$ such that

$$
\eta\left(1+x \pi_{K}^{a}\right)=\operatorname{tr}_{\bar{K} / \mathbb{F}_{p}}\left(x w_{\eta}\right) .
$$

5.4.1. Example. Continuing with the setup in Example 5.3.2, we fix the generator $z$. Fixing the isomorphism $K \cong \bar{K}((\pi))$, we have

$$
\Omega_{\mathcal{O}_{K}}^{1} \otimes_{\mathcal{O}_{K}} \bar{K} \cong \Omega_{\bar{K}}^{1} \oplus \bar{K} d \pi \quad \text { and } \quad \Omega_{\mathcal{O}_{K}}^{1}(\log ) \otimes_{\mathcal{O}_{K}} \bar{K} \cong \Omega_{\bar{K}}^{1} \oplus \bar{K} \frac{d \pi}{\pi} .
$$

Let $d \bar{a}$ be the usual differential of $\bar{a}$ in $\Omega \frac{1}{K}$; it is nonzero if $\bar{a}$ is not a $p$ th power in $\bar{K}$. We can in turn view this element in $\Omega_{\mathcal{O}_{K}}^{1} \otimes_{\mathcal{O}_{K}} \bar{K}$ and $\Omega_{\mathcal{O}_{K}}^{1}(\log ) \otimes_{\mathcal{O}_{K}} \bar{K}$ using the direct sum decomposition above.

We can define a natural homomorphism (in fact an isomorphism) $\rho: \operatorname{Gal}(L / K) \rightarrow$ $\mathbb{F}_{p}$ given by $g \mapsto g(z)-z \in \mathbb{F}_{p}$. Such $\rho$ factors through $\operatorname{gr}^{b_{\text {nlog }}(L / K)} G_{K, \text { nlog }}^{\bullet}$ and $\mathrm{gr}^{b_{\log }(L / K)} G_{K, \log }^{\bullet}$. We describe the image of $\rho$ under the refined Artin and Swan conductor homomorphisms as follows.

In case $(1), \operatorname{rar}(\rho)=\pi^{-n-1} n a d \pi$ and $\operatorname{rsw}(\rho)=\pi^{-n}\left(n a \frac{d \pi}{\pi}+d \bar{a}\right)$.

In case $(2), \operatorname{rar}(\rho)=\pi^{-n} d \bar{a}$ and $\operatorname{rsw}(\rho)=\pi^{-n} d \bar{a}$. (They are not literally the same because they live in different spaces.)

One can check that the refined Swan and Artin conductors do not depend on the choice of $z$.

When $\bar{K}$ is perfect, one can also check that the refined Swan conductor homomorphism is in fact an isomorphism. 
5.4.2. Question. When $\bar{K}$ is not perfect, is the refined Swan conductor homomorphism still an isomorphism? What about the analogous refined Artin conductor homomorphism?

5.5. Multi-index filtration for higher dimensional fields. Using the refined Swan conductor, one can naturally associate a multi-index (upper) filtration for an $m$-CDVF $K$ as follows. We will only treat the case with logarithmic ramification filtration and when the last residue field $k_{0}$ is perfect to simplify the notation; one can easily modify the construction to adapt to the general case.

Let $K$ be an $m$-CDVF with the first residue field $k_{m-1}$. Assume the last residue field $k_{0}$ is perfect. We fix a system of local parameters $t_{1}, \ldots, t_{m}$. In this case, we have

$$
\Omega_{\mathcal{O}_{K}}^{1}(\log ) \otimes_{\mathcal{O}_{K}} k_{m-1}=\bigoplus_{i=1}^{m} k_{m-1} \frac{d t_{i}}{t_{i}} .
$$

For $\lambda=\sum_{i=1}^{m} \alpha_{i} \frac{d t_{i}}{t_{i}} \in \Omega_{\mathcal{O}_{K}}^{1}(\log ) \otimes_{\mathcal{O}_{K}} t_{m}^{-i_{m}} k_{m-1}^{\text {alg }}$, we set

$$
\mathbf{v}_{\log }(\lambda)=\min \left\{\mathbf{v}\left(\alpha_{1}\right), \ldots, \mathbf{v}\left(\alpha_{m}\right)\right\} .
$$

This gives a multi-indexed valuation on $\Omega_{\mathcal{O}_{K}}^{1}(\log ) \otimes_{\mathcal{O}_{K}} t_{m}^{-i_{m}} k_{m-1}^{\text {alg }}$ for $i_{m} \in \mathbb{Q}_{>0}$.

We put $\mathbb{Q}_{>\mathbf{0}}^{m}=\left\{\mathbf{i} \in \mathbb{Q}^{m} \mid i_{m}>0\right\}$. For $\mathbf{i}=\left(i_{1}, \ldots, i_{m}\right) \in \mathbb{Q}_{>\mathbf{0}}^{m}$, we can define a filtration on $G:=G_{K}$ by the following characterization:

$G_{\log }^{\mathbf{i}}:=\left\{g \in G_{\log }^{i_{m}} \mid \chi(g)=0\right.$ for all $\chi: \operatorname{gr}^{i_{m}} G_{\log }^{\bullet} \rightarrow \mathbb{F}_{p}$ such that $\left.\mathbf{v}_{\log }(\operatorname{rsw}(\chi))>-\mathbf{i}\right\}$.

5.5.1. Question. When $K$ has finite last residue field, does this multi-index filtration on $G_{K}^{\mathrm{ab}}$ agree with the one defined by (12) (with $l=1$ ) using the Milnor K-group $K_{n}^{M}(K)$ ? This amounts to comparing the refined Swan conductor homomorphism with the one defined by Kato for characters of $G_{K}^{\text {ab }}$. The comparison is expected by experts. In the equal characteristic case, this is proved in [AS09] and also appears implicitly in Chiarellotto and Pulita $[\mathrm{ChP}]$. But in the mixed characteristic, to our best knowledge, it does not seem to have appeared in the literature.

5.6. A generalization of the theorem of Deligne. Recall from Subsection 3.10 that one expects to be able to associate quotient of Galois groups to truncated discrete valuation rings. More concretely, consider two complete discrete valuation fields $K$ and $K^{\prime}$ and assume that there exists $b \in \mathbb{N}$ such that there is an isomorphism $\mathcal{O}_{K} / \pi_{K}^{b} \mathcal{O}_{K} \cong \mathcal{O}_{K^{\prime}} / \pi_{K^{\prime}}^{b} \mathcal{O}_{K^{\prime}}$ as rings. Unlike in Subsection 3.10, we do not assume that the residue field $\bar{K}=\bar{K}^{\prime}$ is perfect.

5.6.1. Question. Does this isomorphism of rings still imply that $G_{K} / G_{K, \text { nlog }}^{b} \cong$ $G_{K^{\prime}} / G_{K^{\prime}, \log }^{b}$ and $G_{K} / G_{K, \log }^{b} \cong G_{K^{\prime}} / G_{K^{\prime}, \log }^{b}$ ? Are these isomorphisms of quotient groups canonical? Moreover, are they compatible with the refined Swan/Artin conductor homomorphisms?

In the non-logarithmic case, it appears that T. Hiranouchi and Y. Taguchi [HT] have started a project towards proving the isomorphism of quotients of Galois groups. See also the survey paper [Hi].

\section{Three REALIZATIONS OF RAMIFICATION THEORY}

In this section, we discuss three analogous objects that all carry the feature of ramification theory: lisse $l$-adic sheaves, overconvergent $F$-isocrystals, and vector 
bundles with flat connections. In the first two situations, we focus on how AbbesSaito ramification invariants appear geometrically. In the last situation, we focus on the irregularities which are analogs of the Swan conductors in the first two situations.

6.1. $l$-adic representations and lisse $l$-adic sheaves. To start, we first assume that $K$ is of equal characteristic and satisfies the following condition:

(Geom) There exist a smooth scheme $X$ over a field $k$ and an irreducible divisor $D$ smooth over $k$ with the generic point $\eta$, such that $K$ is isomorphic to the completion of $k(X)$ with respect to the valuation given by $\eta$.

Properties for general equal characteristic $K$ may be reduced to the case with this condition by taking certain limit.

Now, given a finite dimensional irreducible $l$-adic representation $\rho$ of $G_{K}$, we may realize it as an $l$-adic sheaf $\mathcal{F}=\mathcal{F}_{\rho}$ over $U:=X-D$, possibly after shrinking $X$. Using vanishing cycles, Saito [Sa09] gave a construction that can detect the highest logarithmic ramification break $b:=b_{\log }(\rho)$. We review this construction here.

Let $\mathcal{I}_{D}$ denote the ideal sheaf for the closed immersion $D \subset X$. Let $(X \times X)^{\prime}$ be the blow-up of $X \times X$ along $D \times D$. Let $(X \times X)^{\sim}$ denote the complement of the proper transform of $(X \times D) \cup(D \times X)$. Let $\tilde{u}:(X \times X)^{\sim} \rightarrow X$ denote the natural projection to the first factor. The diagonal embedding $U \rightarrow U \times U \subset(X \times X)^{\sim}$ extends to a natural embedding $\tilde{\delta}: X \rightarrow(X \times X)^{\sim}$. Let $\mathcal{J}_{X}$ denote the ideal sheaf for this closed immersion. Let $\tilde{j}: U \times U \rightarrow(X \times X)^{\sim}$ denote the natural inclusion.

For $a \in \mathbb{Q}_{\geq 0}$, we use $(X \times X)^{(a)}$ denote the normalization of the scheme associated to the quasi-coherent sub- $\mathcal{O}_{(X \times X) \sim-m o d u l e s}$

$$
\sum_{n \in \mathbb{N}} \tilde{u}^{*}\left(\mathcal{O}_{X}(\lfloor n a\rfloor D)\right) \cdot \mathcal{J}_{X}^{n} \subset \tilde{j}_{*} \mathcal{O}_{U \times U} . \quad \text { (Here, }\lfloor\cdot\rfloor \text { is the floor function). }
$$

When $a$ is a positive integer, this is one of the open charts for the blow-up of $(X \times X)^{\sim}$ along the ideal sheaf $\tilde{u}^{*}\left(\mathcal{I}_{D}\right)^{a}+\mathcal{J}_{X}$.

We use the following notation for morphisms:

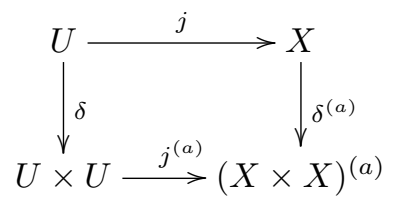

Put $\mathcal{H}:=\mathcal{H} o m\left(\operatorname{pr}_{2}^{*} \mathcal{F}, \operatorname{pr}_{1}^{*} \mathcal{F}\right)$. Saito [Sa09] proved that

6.1.1. Proposition. The highest log ramification break $b_{\log }(\rho) \leq a$ if and only if the base change map

$$
\delta^{(a) *} j_{*}^{(a)} \mathcal{H} \rightarrow j_{*} \mathcal{E} n d(\mathcal{F})
$$

is an isomorphism at the generic point $\eta$ of $D$.

When the condition of the proposition is satisfied, the restriction of $j_{*}^{(a)} \mathcal{H}$ on the complement $(X \times X)^{(a)} \backslash(U \times U)$ is a direct sum of the Artin-Schreier sheaves defined by certain linear forms. These linear forms give rise to the refined Swan conductor homomorphism. 
When the field $K$ is of mixed characteristic, Saito [Sa12] imitates the equal characteristic construction to make sense of $X \times_{k} X$ using infinitesimal deformation. It would be interesting to see if one can put Saito's construction in a more global setting for complete regular rings of mixed characteristic, and obtain global results similar to that in [Sa09].

6.2. $p$-adic differential equations and overconvergent $F$-isocrystals. We first consider the case when $K=\bar{K}((\pi))$ is of equal characteristic and $\bar{K}$ is perfect. Put $F=W(\bar{K})\left[\frac{1}{p}\right]$. Consider the following bounded Robba ring, for $r \in(0,1) \cap p^{\mathbb{Q}}$ :

$$
\mathcal{R}_{\mathrm{bdd}}^{r}:=\left\{\sum_{n \in \mathbb{Z}} a_{n} T^{n}\left|a_{n} \in F,\right| a_{i} \mid \text { is bounded, and } \lim _{i \rightarrow-\infty}\left|a_{i}\right| \cdot r^{i}=0\right\} .
$$

It is the ring of analytic functions on the annulus $r \leq T<1$ which take bounded values.

Let $V$ be an irreducible $p$-adic representation of $G=G_{K}$ with finite image. The theory of Fontaine (see, e. g., [Ke05, Section 4]) associate $V$ with a differential module over $\mathcal{R}_{\mathrm{bdd}}^{r}$ for some positive rational number $r$ sufficiently close to 0 , that is a finite free module $\mathcal{F}=\mathcal{F}_{V}$ over $\mathcal{R}_{\text {bdd }}^{r}$ equipped with a connection

$$
\nabla: \mathcal{F} \rightarrow \mathcal{F} \otimes_{\mathcal{R}_{\text {bdd }}^{r}} \Omega_{\mathcal{R}_{\text {bdd }}^{r} / F}^{1}
$$

This is equivalent to give a derivation $\partial=\frac{d}{d T}$ on $\mathcal{F}$ satisfying Leibniz rule.

This construction allows one to use the full power of the theory of $p$-adic differential equations to the study of ramification theory of $G$. For $r^{\prime} \in p^{\mathbb{Q}}$ with $r^{\prime} \in[r, 1)$, we use $F(T)^{\left(r^{\prime}\right)}$ to denote the completion of $F(T)$ with respect to the $r^{\prime}$-Gauss norm, that is the norm extending the following norm $|\cdot|_{\left(r^{\prime}\right)}$ on $F[T]$ :

$$
\left|\sum_{n \geq 0} a_{n} T^{n}\right|_{\left(r^{\prime}\right)}=\max _{n \geq 0}\left\{\left|a_{n}\right| r^{\prime n}\right\} .
$$

We pick a norm $|\cdot|_{\mathcal{F},\left(r^{\prime}\right)}$ on $\mathcal{F}^{\left(r^{\prime}\right)}:=\mathcal{F} \otimes_{\mathcal{R}_{\mathrm{bdd}}^{r}} F(T)^{\left(r^{\prime}\right)}$ and consider the spectral norm

$$
|\partial|_{\mathrm{sp}, \mathcal{F},\left(r^{\prime}\right)}:=\lim _{n \rightarrow \infty}\left|\partial^{n}\right|_{\mathcal{F},\left(r^{\prime}\right)}^{1 / n},
$$

where $\left|\partial^{n}\right|_{\mathcal{F},\left(r^{\prime}\right)}$ is the operator norm of $\partial^{n}$. The spectral norm does not depend on the chosen norm $|\cdot|_{\mathcal{F},\left(r^{\prime}\right)}$ on $\mathcal{F}^{\left(r^{\prime}\right)}$. This is one of the key invariants for a $p$-adic differential equation. It was explained by Kedlaya in [Ke05] (based on the work of Christol-Mebkhout, Crew, Matsuda, Tsuzuki) that the highest ramification break $b(V)$ has the following characterization by spectral norms:

$$
\text { for } r^{\prime} \text { sufficiently close to } 1^{-},|\partial|_{\mathrm{sp}, \mathcal{F},\left(r^{\prime}\right)}=p^{-1 /(p-1)} \cdot\left(r^{\prime}\right)^{-b(V)-1} \text {. }
$$

A generalization of this approach without the perfectness of $\bar{K}$ was introduced by Kedlaya in [Ke07]. Assume that $\bar{K}$ has a finite $p$-basis (as the general case reduces to this case). The construction works formally the same except the following changes:

- The field $F$ is taken to be the fraction field of a Cohen ring of $\bar{K}$; here the Cohen ring is an absolutely unramified complete discrete valuation ring with residue field $\bar{K}$; we refer to [Wh] for a functorial construction of Cohen rings. 
- We have the derivation $\partial_{0}=\frac{d}{d T}$ as well as other derivations $\partial_{1}, \ldots, \partial_{n}$ coming from a chosen $p$-basis of $\frac{d T}{K}$. Using this, Kedlaya defined a logarithmic differential ramification filtration such that for $r^{\prime}$ sufficiently close to $1^{-}$,

(13) $\max \left\{\left|\partial_{0}\right|_{\mathrm{sp}, \mathcal{F},\left(r^{\prime}\right)} \cdot r^{\prime},\left|\partial_{1}\right|_{\mathrm{sp}, \mathcal{F},\left(r^{\prime}\right)}, \ldots,\left|\partial_{n}\right|_{\mathrm{sp}, \mathcal{F},\left(r^{\prime}\right)}\right\}=p^{-1 /(p-1)} \cdot\left(r^{\prime}\right)^{-b_{\log }(V)}$, where, as before, $b_{\log }(V)$ is the highest ramification break defined by the differential ramification filtration.

A different normalization of (13) by removing the factor $r^{\prime}$ in the first term gives rise to a non-log ramification filtration. Moreover, Kedlaya proved that his differential ramification filtration has the analogous Hasse-Arf property using the integrality of Newton polygons. (One can also deduce this by reducing to the perfect residue field case.)

It is proved in [X10] that Kedlaya's differential filtration agrees with Abbes and Saito filtration; this then proves Theorem 5.3.3 in the equal characteristic by transferring the Hasse-Arf property through the comparison.

Moreover, in the equal characteristic case, $[\mathrm{X} 12 \mathrm{~b}]$ realizes the refined Swan conductor homomorphism using $p$-adic differential modules; this is related to the eigenvalues of the matrices for the differential operators $\partial_{0}, \ldots, \partial_{n}$, acting on an appropriate basis of $\mathcal{F}$. [X12b] further relates the refined Swan conductor homomorphism to the variation of Swan conductor (see Subsection 10.2).

When $K$ is of mixed characteristic under some mild condition, it is proved in [X12a] that one can "fake" the Robba ring construction above and apply the recent results $[\mathrm{Ke} 10 \mathrm{a}, \mathrm{KeX}]$ on $p$-adic equation equations to deduce the Hasse-Arf theorem.

6.2.1. Question. Can we realize the refined Swan conductor homomorphism in the mixed characteristic case, using the fake Robba ring construction?

6.3. Vector bundles with irregular singularities. The object analogous to representations of $G_{K}$ (when $\bar{K}$ is perfect) is a differential module over $\mathbb{C}((T)$ ), that is a finite dimensional vectors space $V$ over $\mathbb{C}((T))$ equipped with a derivation $\partial=\frac{d}{d T}$ (satisfying the Leibniz rule). Such a module is called regular if $T \partial$ preserves a $\mathbb{C} \llbracket T \rrbracket$-lattice $\Lambda$ of $V$. For $P \in \mathbb{C}((T))$, we can define a rank one module $E(P)=$ $\mathbb{C}((T)) \cdot e$ such that $\partial(e)=P e$.

The Turrittin-Levelt-Hukuhara Theorem (see, e. g., [Ke10a, Section 7.5]) says that there exists $n \in \mathbb{N}$ such that we have a decomposition

$$
V \otimes_{\mathbb{C}((T))} \mathbb{C}\left(\left(T^{1 / n}\right)\right) \cong \oplus_{i=1}^{r} V_{i},
$$

where each $V_{i}$ is of the form $V_{i}=E\left(P_{i}\right) \otimes R_{i}$ for an element $P_{i} \in \mathbb{C}\left(\left(T^{1 / n}\right)\right)$ and a regular module $R_{i}$ over $\mathbb{C}\left(\left(T^{1 / n}\right)\right)$.

The analogous invariant of ramification break is just $\max \left\{0,-v_{\mathbb{C}((T))}\left(P_{i}\right)\right\}$. We define the irregularity of $V$ to be

$$
\operatorname{Irr}(V):=\sum_{i=1}^{r} \operatorname{dim} V_{i} \cdot \max \left\{0,-v_{\mathbb{C}((T))}\left(P_{i}\right)\right\} .
$$

We can give an interpretation of this invariant in terms of the spectral norms of the differential operators $\partial$. For details, see [Ke10a, X12b].

In the general case when $K=\bar{K}((T))$ with $\bar{K}$ has characteristic zero, there might be additional derivations $\partial_{1}, \ldots, \partial_{n}$ on $\bar{K}$. For example, when $\bar{K}=\mathbb{C}(x, y)$, we may consider the derivations $\partial_{1}=\frac{d}{d x}$ and $\partial_{2}=\frac{d}{d y}$. We consider a differential module 
$V$ over $\bar{K}((T))$, that is a finite dimensional vector space $\bar{K}((T))$ equipped with commuting actions of $\partial_{0}=\frac{d}{d T}, \partial_{1}, \ldots, \partial_{n}$. When $V$ is irreducible, one can define the irregularity of $V$ by taking the maximum among all irregularities computed by the spectral norms of all differential operators. For general $V$, its irregularity is defined to be the sum of the irregularities over all Jordan-Hölder constituents. For the details, we refer to $[\mathrm{X} 12 \mathrm{~b}]$.

Similarly, one can define a refined irregularity as an analogue of the refined Swan conductor for Galois representations. This is also explained in [X12b].

\section{Elimination theORY}

7.1. The expectations. We see that the Kato-Swan conductor as well as the ramification filtration of Abbes-Saito work perfectly in all the situations where one needs the ramification invariants that "live downstairs", i. e., for an extension $L / K$, are more closely attached to $K$ than to $L$. These include multiple questions related to the absolute Galois group of a complete discrete valuation field, or, in algebraic geometry, to the étale site of an algebraic or arithmetic variety.

In other words, we have the best possible "upper ramification filtration" 3 . However, in general we cannot recover the usual (lower) ramification filtration from it, there are no Hasse-Herbrand functions, and we cannot write down any analogs of the functorial properties in Subsections 3.2, 3.3, 3.4, 3.5. The reason for this is rather fundamental: any single ramification filtration as well as any theory of Swan-type conductor describes the ramification of an extension of degree $p$ with just one number. But we saw in the example in section 4 that the "comprehensive" ramification theory should provide more information in this case. Indeed, in (11) we have to know not only $n$ and $N$ but also $m$.

Also, we have no formula for the order of different (or depth) ${ }^{4}$ in terms of upper breaks which would be a substitute for (10). The best possible estimates in the case of an $n$-dimensional local field (with finite last residue field) are given by Hyodo inequalities (see $[\mathrm{Hy}]$, Th. (1-5), Prop. (3-4), Ex. (3-5)):

$$
(p-1) \sum_{l \geq 1} \frac{\mathbf{j}_{L / K}(l)}{p^{l}} \leq \mathbf{d}_{K}(L / K) \leq \frac{p-1}{p} \sum_{l \geq 1} \mathbf{j}_{L / K}(l),
$$

where $\mathbf{j}_{L / K}(l)$ are defined in (12).

A possible distant goal for further investigation of ramification in the imperfect residue field case could be to construct a certain system of invariants $\Sigma(L / K)$ for any finite extension $L / K$ which would completely describe the ramification of $L / K$. This vague desire can be made more specific by listing at least the following requirements.

(1) "Naïve" ramification invariants (ramification index, order of different, genome, Artin and Swan ramification numbers) as well as other important invariants (such as Abbes-Saito conductor) can be expressed in terms of $\Sigma(L / K)$.

(2) Ramification of intermediate extensions (i. e., $\Sigma(L / M)$ and $\Sigma(M / K)$ ) can be expressed in terms of $\Sigma(L / K)$; reasonable base change properties in spirit of Prop. 3.3.2 are available.

\footnotetext{
${ }^{3}$ The terminology is absolutely misleading! The upper ramification breaks live downstairs, and the lower ones live upstairs.

${ }^{4}$ The order of different and the depth can be considered as invariants that "live in the middle".
} 
(3) Local terms of appropriate global formulas can be expressed in terms of $\Sigma(L / K)$.

7.2. Background. Here we discuss a theory producing some additional ramification information that can be organized in analogs of lower and upper filtrations. The approach, with the origin in [Ka87], is based on two observations.

1. The Herbrand theorem (5) is true not only in the classical case but, more generally, in all the monogenic cases, i. e., when $\mathcal{O}_{L}=\mathcal{O}_{K}[x]$ for some $x$. Consequently, the ramification invariants of monogenic extensions, defined in a usual manner, possess all the usual functorial properties. The inverse statement is also true; more precisely ([Sp99, Prop. 1.5.2]):

7.2.1. Proposition. Let $L / K$ be a finite Galois p-extension. Then the following properties are equivalent:

(i) $\mathcal{O}_{L}=\mathcal{O}_{K}[x]$ for some $x$;

(ii) for every normal subgroup $H$ of $G$ the Herbrand property (5) holds;

(iii) the Hilbert formula holds:

$$
v_{L}\left(\mathcal{D}_{L / K}\right)=\sum_{\sigma \neq 1} i_{G}(\sigma)=\sum_{i \geq 0}\left(\left|G_{i}\right|-1\right) .
$$

In [Sp99] such extensions are called well ramified. There are three types of well ramified extensions.

Case I. All the extensions with separable $\bar{L} / \bar{K}$.

Case II. All the weakly unramified extensions such that $\bar{L} / \bar{K}$ is generated by 1 element. (In particular, if $K$ is a two-dimensional local field, or, more generally, if $\left[\bar{K}: \bar{K}^{p}\right]=p$, then all weakly unramified extensions of $K$ are well ramified.)

Case III. Those well ramified extensions that belong neither to Case I nor to Case II. Spriano showed that for any $L / K$ from Case III there exists an intermediate field $M$ such that $M / K$ is in Case I, and $L / M$ is in Case II. A general description of Case III extensions was given in [HLF, Sect. I, §18] and [Sp00].

For us, the above remark on two-dimensional fields is important.

2. Let $L / K$ be any finite Galois extension of complete discrete valuation fields with imperfect residue fields of characteristic $p>0$, and let $k$ be a constant subfield of $K$, i. e., a maximal complete subfield of $K$ with perfect residue field. (If char $K=$ 0 , such a subfield is unique.) Epp's theorem on elimination of wild ramification $[\mathrm{E}]$ (corrections in $[\mathrm{P}]$ and [Kuhl]) asserts that there exists a finite extension $k^{\prime} / k$ such that $k^{\prime} L / k^{\prime} K$ is weakly unramified. The paper [KZ] contains various refined versions of Epp's theorem, with application to classification of higher local fields.

7.3. Construction. Now we are ready to describe the construction from [Z03] and [HLF, Sect. I, §17]. For a given complete two-dimensional ${ }^{5}$ discrete valuation field $K$, fix a constant subfield $k$. An extension $L / K$ is said to be constant if $L=k^{\prime} K$ and almost constant, if $L \subset k^{\prime} K_{u}$, where $k^{\prime} / k$ is a finite extension, and $K_{u} / K$ is an unramified extension. We say that a field $L$ is standard if a prime element of its constant subfield is also a prime element of $L$. The choice of a constant subfield $k$ in $K$ determines a constant subfield $l$ in $L$ which is algebraic over $k$.

5. e. such that $\left[\bar{K}: \bar{K}^{p}\right]=p$ 
For any finite Galois extension $L / K$ denote by $L_{0}$ the inertia subfield in $L / K$ and by $L_{c} / K$ the maximal almost constant subextension in $L / K$. The idea is to induce:

(1) the ramification filtration on $\operatorname{Gal}\left(L_{c} / L_{0}\right)$ by the filtration for the corresponding constants subfields;

(2) the ramification filtration on $\operatorname{Gal}\left(L / L_{c}\right)$ by the filtration on an isomorphic group $\operatorname{Gal}\left(k^{\prime} L / k^{\prime} L_{c}\right)$, where $k^{\prime} / k$ is a finite extension that makes $\operatorname{Gal}\left(k^{\prime} L / k^{\prime} L_{c}\right)$ weakly unramified by Epp's theorem (and even ferocious in view of the definition of $\left.L_{c}\right)$.

Namely, introduce a set

$$
\mathbb{I}=\{-1,0\} \cup\{(\mathbf{c}, i) \mid i \in \mathbb{Q}, i>0\} \cup\{(\mathbf{c}, \infty)\} \cup\{(\mathbf{i}, i) \mid i \in \mathbb{Q}, i>0\}
$$

with linear order

$$
\begin{aligned}
& -1<0<(\mathbf{c}, i)<(\mathbf{i}, j) \\
& \text { for any } i, j \text {; } \\
& (\mathbf{c}, i)<(\mathbf{c}, j) \\
& \text { for any } i<j \text {; } \\
& (\mathbf{i}, i)<(\mathbf{i}, j) \\
& \text { for any } i<j \text {. }
\end{aligned}
$$

This will be the index set for lower and upper numbering of new ramification subgroups.

Let $G=\operatorname{Gal}(L / K)$. We put $G_{-1}=G$, and denote by $G_{0}$ the usual inertia subgroup in $G$.

To introduce subgroups $G_{(\mathbf{c}, i)}=G_{\mathbf{c}, i}$, we consider first the case when $L_{c} / K$ is constant and contains no unramified subextension. Then $L_{c}=l K$, and we have a natural projection

$$
p: \operatorname{Gal}(L / K) \rightarrow \operatorname{Gal}\left(L_{c} / K\right)=\operatorname{Gal}(l / k)=\operatorname{Gal}(l / k)_{0} .
$$

Then we put $G_{\mathbf{c}, i}=p^{-1}\left(\operatorname{Gal}(l / k)_{i}\right)$. In the general case take an unramified extension $K^{\prime} / K$ such that $K^{\prime} L / K^{\prime}$ contains no unramified subextension, and the maximal almost constant subextension in $K^{\prime} L / K^{\prime}$ (i. e., $K^{\prime} L_{c} / K^{\prime}$ ) is constant. We put $G_{\mathbf{c}, i}=\operatorname{Gal}\left(K^{\prime} L / K^{\prime}\right)_{\mathbf{c}, i}$. Next,

$$
G_{\mathbf{c}, \infty}=\operatorname{Gal}\left(L / L_{c}\right)=G_{\mathbf{c}, m}
$$

for $m$ big enough.

Assume that $L_{c}$ is standard and $L / L_{c}$ is ferocious. Let $t \in \mathcal{O}_{L}, \bar{t} \notin \bar{L}^{p}$. We define

$$
G_{\mathbf{c}, i}=\left\{g \in \operatorname{Gal}\left(L / L_{c}\right) \mid v_{K}(g(t)-t) \geq i\right\}
$$

for all $i>0$.

In the general case choose a finite extension $l^{\prime} / l$ such that $l^{\prime} L_{c}$ is standard and $e\left(l^{\prime} L / l^{\prime} L_{c}\right)=1$; this is possible by Epp's theorem. Then $\operatorname{Gal}\left(l^{\prime} L / l^{\prime} L_{c}\right)=$ $\operatorname{Gal}\left(L / L_{c}\right)$, and $l^{\prime} L / l^{\prime} L_{c}$ is ferocious. We define

$$
G_{\mathbf{c}, i}=\operatorname{Gal}\left(l^{\prime} L / l^{\prime} L_{c}\right)_{\mathbf{c}, i}=\operatorname{Gal}\left(l^{\prime} L / l^{\prime} K\right)_{\mathbf{c}, i}
$$

for all $i>0$; these groups are independent of the choice of $l^{\prime}$ since we used $v_{K}$ (and not $\left.v_{L}\right)$ in (15).

This gives a well defined lower ramification filtration on $G$ indexed by $\mathbb{I}$; one can define Hasse-Herbrand functions from $\mathbb{I}$ to $\mathbb{I}$ with usual properties and, consequently, construct the upper filtration. The compatibility with subgroups and factor groups 
mimics that of the classical case, and a ramification filtration for infinite Galois extensions is defined.

One can note also that we obtained filtration (on finite Galois groups) which factors $G_{i} / G_{i+}$ are abelian for $i \geq 0$ (even elementary abelian for $i>0$ ). This would not be true if we did not consider the contribution of c-part. For a 2dimensional local field, one could also define a refined $\mathbb{I}_{2}$-filtration using rank 2 valuations in the i-part $([\mathrm{Z03}, \S 4])$.

7.4. Further properties. There exists also a partial result on compatibility with the higher class field theory. Namely, for an equal characteristic 2-dimensional local field $K$ with finite residue field, one can define explicitly an $\mathbb{I}_{2}$-filtration on $K_{2}^{\text {top }} K$ which coincides with the inverse image of the ramification filtration on $\operatorname{Gal}\left(K^{\mathrm{ab}} / K\right)$, see $[Z 03, \S 6]$.

It is not so easy to do the same in the mixed characteristic case because of the more complicated $\operatorname{Gal}\left(K^{\mathrm{ab}} / K\right)$ and the presence of $p$-torsions in $K_{2}^{\mathrm{top}} K$. In particular, the following question is of interest.

\subsubsection{Question. What is $C_{K}=\Theta_{K}^{-1}\left(\operatorname{Gal}\left(K^{\mathrm{ab}} / K_{c}^{\mathrm{ab}}\right)\right)$ ?}

By the results of Miki [Mik74], any extension of $K$ with the Galois group $\mathbb{Z}_{p}$ is almost constant. This means that $K^{\Gamma} / K$, the compositum of all $\mathbb{Z}_{p}$-extensions, is a subextension of $K_{c}^{\mathrm{ab}} / K$. On the other hand, $K_{c}^{\mathrm{ab}}=k^{\mathrm{ab}} K^{\mathrm{ab} \text {,ur }}=k^{\mathrm{ab}} K^{\mathrm{ab} \text {,tr }}$, and it is easy to see that $K^{\Gamma} K^{\mathrm{ab}, \mathrm{tr}}=k^{\Gamma} K^{\mathrm{ab} \text {,tr }}$, where $K^{\mathrm{ab}, \mathrm{ur}} / K\left(\operatorname{resp} . K^{\mathrm{ab}, \mathrm{tr}} / K\right)$ is the maximal abelian unramified (resp. tamely ramified) extension of $K$. Therefore,

$$
\begin{aligned}
\operatorname{Gal}\left(K_{c}^{\mathrm{ab}} / K^{\Gamma} K^{\mathrm{ab}, \operatorname{tr}}\right) & =\operatorname{Gal}\left(k^{\mathrm{ab}} K^{\mathrm{ab}, \operatorname{tr}} / k^{\Gamma} K^{\mathrm{ab}, \mathrm{tr}}\right) \\
& \simeq \operatorname{Gal}\left(k^{\mathrm{ab}} / k^{\Gamma} k^{\mathrm{ab}, \operatorname{tr}}\right) \\
& \simeq \text { torsions in } U_{1, k}
\end{aligned}
$$

by usual local class field theory.

Let $T_{K}$ be the topological closure of $p$-torsions in $K_{2}^{\text {top }} K$. Since there is no $p$-torsion in $K^{\Gamma} K^{\mathrm{ab}, \mathrm{tr}} / K$, we have $\Theta_{K}\left(T_{K}\right) \subset \operatorname{Gal}\left(K^{\mathrm{ab}} / K^{\Gamma} K^{\mathrm{ab}, \mathrm{tr}}\right)$. From the explicit description of generators of $K_{2}^{\mathrm{top}} K / T_{K}$ (see [Z97], [I08]), it is clear that even $\Theta_{K}\left(T_{K}\right)=\operatorname{Gal}\left(K^{\mathrm{ab}} / K^{\Gamma} K^{\mathrm{ab}, \mathrm{tr}}\right)$. This means that $C_{K}$ should be a subgroup of index $p^{m}$ in $T_{K}$, where $p^{m}$ is the order of $p$-torsions in $k^{*}$ (or in $K^{*}$ ). However, what are the generators of $C_{K}$ ?

The above described ramification filtration gives a way of generalizing the "anabelian yoga" to higher local fields. Abrashkin [Abr02] generalized the above construction from 2 -dimensional case to $n$-dimensional local fields, introducing ramification theory that depends on the choice of $i$-dimensional subfields $K_{i}(1 \leq i \leq$ $n-1)$ in the given $n$-dimensional local field, and proved a complete analog of his 1-dimensional result (announced in [Abr02], full proof in the equal characteristic 2-dimensional case in [Abr03]).

Next, Abrashkin used his generalized ramification theory to develop an analog of the functor of field of norms for higher dimensional local fields, see [Abr07]. Note that there exists further generalization of the field of norms functor to the case of arbitrary imperfect residue field with finite $p$-basis by A. J. Scholl [Sch]; his construction does not use any kind of higher ramification theory.

Despite these nice properties, the $\mathbb{I}$-ramification theory is quite far from being a "Traumverzweigungstheorie". In particular, even for an extension of prime degree 
its I-ramification break does not determine its depth of ramification and even its genome ("W" or "F"). For example, let $K=F((t))((\pi))$ and $k=F((\pi)), F$ being a finite field. Assume that $L / K$ corresponds to the Artin-Schreier equation $x^{p}-x=\pi^{-n}+t \pi^{-p m}$, where $m, n$ are positive integers. Then the $\mathbb{I}_{2}$-break of $L / K$ is $m$ for any $n$, whereas $d_{K}(L / K)=\frac{p-1}{p} \max \{n, p m\}$, and $L / K$ is wild iff $n \geq p m$.

However, in the equal characteristic case one can vary the constant subfield $k$ of $K$ thus collecting more information on ramification. For example, if $L / K$ is wild of degree $p$ with the Swan number $s_{0}$, then, for some choices of $k$, the $\mathbb{I}$-break of $L / K$ is $(\mathbf{c}, s)$ and necessarily $s=s_{0}$. In this example $m$ is not an invariant of $L / K$. However, if in the example of $\S 4$ we consider only such $k$ that the $\mathbb{I}$-break of $K_{2} / K$ is some $(\mathbf{c}, s)$ (clearly, $s=N)$, then the $\mathbb{I}$-break of $K_{1} / K$ will be $(\mathbf{i}, m / p)$. Therefore, the knowledge of $\mathbb{I}$-breaks of $K_{1} / K$ and $K_{2} / K$ for all choices of $k$ determines the ramification of $K_{1} K_{2} / K$.

7.4.2. Question. Can we construct a powerful ramification theory for equal characteristic 2-dimensional fields by varying the constant subfield?

7.4.3. Question. Can we use this approach even in the mixed characteristic case using truncations from [De84]?

\section{Semi-GLOBAl MODELling}

Now we describe one more approach to description of ramification in the imperfect residue field case. This approach goes back to P. Deligne who sketched a proof of Grothendieck-Ogg-Shafarevich formula for surfaces in his famous letter to L. Illusie [De76].

8.1. Background. We recall some starting points of Deligne's program. Let $\mathcal{F}$ be a locally constant étale $\mathbb{F}_{l}$-sheaf of finite rank on $U$, where $U$ is the complement to some divisor $D$ on a smooth projective surface $S$ over an algebraically closed field of prime characteristic $p \neq l$. In order to understand the ramification data associated with $\mathcal{F}$ at the generic point of a component $D_{0}$ of $D$, Deligne considers various regular $\operatorname{arcs} \mathcal{C}$ transversal to $D_{0}$ and studies the restrictions of $\left.\mathcal{F}\right|_{\mathcal{C}}$ to these arcs. It is expected that the Swan conductor of $\left.\mathcal{F}\right|_{\mathcal{C}}$ (at the point where $\mathcal{C}$ meets $D_{0}$ ) depends only on the jet of $\mathcal{C}$ of certain order $r$. Thus, we can consider the Swan conductor as a function on the space $T_{1, r}$ of $r$-jets of arcs transversal to $D_{0}$; this space has a natural structure of vector bundle over $D_{0}$. Next, this function is expected to be lower semi-continuous; in particular, it should take its maximal value over certain Zariski open subset $W$ of $T_{1, r}$. The next claim is that the complement of $W$ has pure codimension one in $T_{1, r}$, i. e., is a union of several irreducible hypersurfaces. The further work is based on geometry of these hypersurfaces including intersection theory.

Some of these facts were proved in [La] under assumption of "absence of ferocious ramification". This means that the locally constant sheaf $\mathcal{F}$ is trivialized in some finite extension of $k(S)$ such that all extensions of residue fields are separable. In particular, the semi-continuity of Swan conductor has been proved under this assumption.

Brylinski in $[\mathrm{Br}]$ considers a cyclic $p$-extension of the function field of s surface $S$ over a field of characterstic $p$ given by the Witt vector $x=\left(x_{0}, \ldots, x_{r-1}\right)$. He assumes that the branch locus $D_{0}$ is smooth at a certain regular point $P$ of $S$ and the valuations of all $x_{i}$ at the generic point of $D_{0}$ are either positive or prime to 
p. (This condition implies absence of ferocious ramification if $r=1$ but not in general.) Under this assumption he proves that, for all curves $C$ transversal to $D_{0}$ at $P$, the Swan conductors of corresponding extensions of $k(C)$ are equal, and their common value is Kato-Swan conductor of the extension of the 2-dimensional local field $k(S)_{D_{0}, P}$ corresponding to $x$.

Consider a cyclic extension $L$ of degree $p$ of $k(S)$ as above such that the branch locus $D_{0}$ is smooth with one component, and the ramification at this component is wild. We see from the papers of Laumon and Brylinski that in this case for all curves $C$ transversal to $D$ at a fixed point, the corresponding ramification numbers will be the same (and equal to the ramification number of $L / k(S)$ ). However, in order to approach a more comprehensive description of ramification in the sense of Subsection 7.1, it appeared useful to consider curves which are tangent to $D_{0}$ of certain fixed order (and smooth).

8.1.1. Example. Let $k$ be algebraically closed, char $k=2, S=\mathbb{A}_{k}^{2}$ with coordinates $t, u, S^{\prime}$ the normalization of $S$ in the Artin-Schreier extension $L_{\alpha} / k(t, u)$ given by

$$
x^{2}-x=t^{-2 n+1}(1+\alpha u),
$$

where $\alpha \in k$. Introducing $t_{1}=t^{n} x$, wee see that $t_{1}$ is integral over $k[t, u]$ and $S_{0}^{\prime}=$ Spec $k\left[t, u, t_{1}\right]$ is regular, whence $S^{\prime}=S_{0}^{\prime}$. Let $O^{\prime}$ be the closed point of $S^{\prime}$ above the origin $O$. (It is unique since $O$ belongs to the branch locus of normalization morphism.) Replacing $S$ and $S^{\prime}$ with the spectra of completed local rings at $O$ and $O^{\prime}$ respectively, and introducing $t_{0}=t(1+\alpha u)$, we arrive at the morphsim $\varphi: k\left[\left[t_{0}, u\right]\right] \rightarrow k\left[\left[t_{1}, u\right]\right]$ given by

$$
\varphi\left(t_{0}\right)=t_{1}^{2}+t_{1}^{2 n+1}+\text { terms of higher order. }
$$

Notice that the branch locus of $\varphi$ is determined by the prime ideal $\left(t_{0}\right)$ of $k\left[\left[t_{0}, u\right]\right]$. Consider a family of curves $C_{\lambda}$ on Spec $k\left[\left[t_{0}, u\right]\right]$ with the equations

$$
t_{0}=u^{2}+\lambda u^{3}+u^{5}, \quad \lambda \in k,
$$

and denote by $C_{\lambda}^{\prime}$ their pullbacks in Spec $k\left[\left[t_{1}, u\right]\right]$. It is not difficult to calculate that

$$
s\left(k\left(C_{\lambda}^{\prime}\right) / k\left(C_{\lambda}\right)\right)= \begin{cases}4 n-3, & \lambda \neq 0 \\ 4 n-5, & \lambda=0\end{cases}
$$

(assuming $n \geq 2$ ). Moreover, let $C$ be an arbitrary regular curve on $\operatorname{Spec} k\left[\left[t_{0}, u\right]\right]$ which is simply tangent to the branch divisor, i. e., with an equation

$$
t_{0}=\lambda_{2} u^{2}+\lambda_{3} u^{3}+\ldots
$$

where $\lambda_{2} \neq 0$, and let $C^{\prime}$ be its pullback. Then $C^{\prime}$ is irreducible; $s\left(k\left(C^{\prime}\right) / k(C)\right)=$ $4 n-3$ if $\lambda_{3} \neq 0$, and $s\left(k\left(C^{\prime}\right) / k(C)\right)<4 n-3$ if $\lambda_{3}=0$ ("exceptional hypersurface"). Note that if $C$ is determined by an equation

$$
t=\mu_{2} u^{2}+\mu_{3} u^{3}+\ldots
$$

in the original coordinates $t, u$, then $\mu_{3}=\lambda_{3}+\alpha \mu_{2}$. This means that the equation of the "exceptional hypersurface" $H_{\alpha}$ is $\mu_{3}=\alpha \mu_{2}$, and thus $H_{\alpha}$ "detects the $\alpha$ ". 
8.2. Semi-global models. Deligne's program is intended to compute Euler-Poincaré characteristic of an étale sheaf on a surface or, more generally, to describe ramification of a finite morphism of algebraic or arithmetic surfaces. However, we can try to use this approach as a source of rich information about ramification of extensions of 2-dimensional local fields by constructing geometric "models" for given extensions.

Namely, let $h: A \rightarrow B$ be a finite $k$-homomorphism of 2-dimensional regular local rings with perfect coefficient subfield $k$ of characteristic $p>0$. Let $\mathfrak{p}$ be a prime ideal of height 1 in $B$ such that $B / \mathfrak{p}$ and $A / h^{-1}(\mathfrak{p})$ are regular. We shall say that $(h, \mathfrak{p})$ is a model for a finite extension of 2-dimensional local fields $L / K$, if there exists an isomorphism $i$ of 2-dimensional local fields $\widehat{Q(B)}_{\mathfrak{p}} \simeq L$ mapping $\widehat{Q(A)}_{h^{-1}(\mathfrak{p})}$ onto $K$.

We suggest to study ramification in $L / K$ by considering various regular curves on Spec $A$ and their pullbacks in Spec $B$. For each such curve $C$ and a component of its pullback $C^{\prime}$, the field extension $k\left(C^{\prime}\right) / k(C)$ is a finite extension of 1-dimensional local fields inheriting information on $L / K$.

Of course, since we are interested only in "ramification in codimension 1", we have a huge freedom in choosing models for given $L / K$. (We can make blow-ups preserving $L / K$ etc.) We hope to describe a class of morphisms $h$ having as simple structure as possible to make the study of $k\left(C^{\prime}\right) / k(C)$ easy but still providing models for all $L / K$ of interest.

For example, in [Z10] we proposed to study pairs $(h, \mathfrak{p})$ such that for some choice of regular local parameters $t, u$ in $A$ and $x, y$ in $B$ with $\mathfrak{p}=(x)$ and $h^{-1}(\mathfrak{p})=(t)$ the following conditions are satisfied:

(i) $h(t)=\delta \cdot x^{e_{x}}$,

(ii) $h(u) \equiv \varepsilon \cdot y^{e_{y}} \bmod x$,

(iii) $J(t, u)=\left|\begin{array}{ll}\frac{\partial h(t)}{\partial x} & \frac{\partial h(t)}{\partial y} \\ \frac{\partial h(u)}{\partial x} & \frac{\partial h(u)}{\partial y}\end{array}\right|=\gamma \cdot x^{M}$,

where $e_{x}, e_{y}$ are positive integers, $e_{y}$ being a nonnegative power of $p, M$ is a nonnegative integer; $\delta, \varepsilon, \gamma \in B^{*}$.

Such morphisms appeared in $[\mathrm{CuP}]$ in the context of resolution of a finite morphism between regular algebraic surfaces over a field of characteristic $p>0$.

It was proved in [Z10, Prop. 2.4] that an extension of 2-dimensional local fields $L / K$ has a model with properties (i), (ii), if the following 2 conditions are satisfied.

(1) $f_{s}(L / K)=1$.

(2) Let $\left(e_{i j}\right)_{i, j=1,2}$ be the matrix ramification index for some choice of rank 2 valuations $\mathbf{v}_{L}$ and $\mathbf{v}_{K}$, i. e., $\left.\mathbf{v}_{L}\right|_{K}=\mathbf{v}_{K} \cdot\left(e_{i j}\right)$. Then $\operatorname{gcd}\left(e_{11}, e_{22}\right) \mid e_{12}$.

Moreover, in this case we have $e_{w}(L / K)=e_{x}$ and $f_{i}(L / K)=e_{y}$, see [Z10, Prop. $2.2]$.

8.3. Initial questions. Let $(h, \mathfrak{p})$ be as in Subsection 8.2; denote by $D_{0}$ the prime divisor of $X=\operatorname{Spec} A$ corresponding to $h^{-1}(\mathfrak{p})$. Fix a positive integer $r$ and consider the set $T_{r}$ of all regular $\operatorname{arcs} C$ on $X$ such that $\left(C . D_{0}\right)=r$. Assume the above condition (iii); then $C$ is not a component of the branch divisor, and $h^{*} C=C_{1}^{\prime}+\cdots+C_{n}^{\prime}$, where $C_{1}^{\prime}, \ldots, C_{n}^{\prime}$ are distinct prime divisors of $\operatorname{Spec} B$, and $n=n(C)$ is a positive integer. For each $i(1 \leq i \leq n)$, we have an extension of complete discrete valuation fields with perfect residue fields $k\left(C_{i}^{\prime}\right) / k(C)$. Our plan 
is to study the ramification invariants of the extensions $k\left(C_{i}^{\prime}\right) / k(C)$ as functions on the set $T_{r}$.

First of all, we have to check that $n(C)$ and all the ramification invariants depend only on the jet of $C$ of certain order $R=R(r)$. Having this proved, we can consider $n(C)$ and ramification invariants as functions on the set $T_{r, R}$ of $R$-jets of arcs from $T_{r}$.

Each $T_{r, R}$ has a structure of an affine variety over $k$. Indeed, let $t, u$ be local parameters of $A$ such that $(t)=h^{-1}(\mathfrak{p})$. Then, in view of Weierstraß preparation theorem, each curve from $T_{r}$ has a unique equation of the form

$$
f= \begin{cases}-u+\alpha_{1} t+\alpha_{2} t^{2}+\ldots, & r=1, \\ -t+\beta_{r} u^{r}+\beta_{r+1} u^{r+1}+\ldots, & r>1,\end{cases}
$$

where $\alpha_{i}$ and $\beta_{i}$ are any elements of $k$ with an only restriction $\beta_{r} \neq 0$. If $r>1$, $T_{r, R}$ can be identified with $\left\{\left(\beta_{r}, \ldots, \beta_{R}\right) \in \mathbb{A}_{k}^{R-r+1} \mid \beta_{r} \neq 0\right\}$; if $r=1, T_{r, R}$ can be identified with $\mathbb{A}_{k}^{R}$; see more details in [Z02a].

Next, we would like to check that certain functions of these ramification invariants are semi-continuous on $T_{r, R}$ with respect to corresponding Zariski topology. (These functions are reduced to conductors or the order of different if $s=1$, and the precise definitions in the general case is still to be understood.)

Some results in this direction are included into the next section.

\section{Some RESUlts On SEMI-CONTINUity}

9.1. Artin-Schreier extensions. The paper [Z02a] is devoted to the study of questions raised in subsection 8.3 in the case of Artin-Schreier coverings of the spectrum of a complete 2-dimensional regular local ring (of characteristic $p>0$ ). Such coverings can serve as semi-global models of Artin-Schreier extensions of 2dimensional local fields. However, the setting in this work is somewhat more general: the morphisms with 2 (transversal) components in the branch locus are also included into consideration.

More precisely, let $A$ be a regular two-dimensional local ring (not necessarily complete), char $A=p>0, K=Q(A), \mathfrak{m}$ the maximal ideal of $A$, and $k$ the residue field which is assumed to be algebraically closed. For a prime ideal $\mathfrak{p}$ of height 1 , denote by $F_{\mathfrak{p}}$ the corresponding prime divisor of Spec $A$. For any two distinct prime divisors $F_{\mathfrak{p}}, F_{\mathfrak{p}^{\prime}}$ we define their intersection number as

$$
\left(F_{\mathfrak{p}} \cdot F_{\mathfrak{p}^{\prime}}\right)=\operatorname{dim}_{k} A /\left(\mathfrak{p}+\mathfrak{p}^{\prime}\right)
$$

by linearity this definition can be extended to any two divisors $C, D$ with no common components.

Let $L / K$ be a cyclic extension of degree $p$, and let $B$ be the integral closure of $A$ in $L$. For the sake of simplicity of statements we assume here that the branch divisor of $B / A$ consists of one smooth component $F_{\mathfrak{p}_{1}}$; for the case of two transversal components, see [Z02a]. Denote by $U_{A}$ the set of prime ideals of height 1 of $A$ other than $\mathfrak{p}_{1}$. For $\mathfrak{p} \in U_{A}$, denote by $\mathfrak{q}$ any prime ideal of $B$ over $\mathfrak{p}$. Denote

$$
s_{\mathfrak{p}}(L / K)= \begin{cases}s(L(\mathfrak{q}) / K(\mathfrak{p})), & e(L(\mathfrak{q}) / K(\mathfrak{p}))=p \\ 0, & \text { otherwise }\end{cases}
$$

where $K(\mathfrak{p})$ is the fraction field of $A / \mathfrak{p}$, and $L(\mathfrak{q})$ is the fraction field of $B / \mathfrak{q}$.

Introduce $T_{r}$ and $T_{r, n}$ as in Subsection 8.3 and identify $\mathfrak{p}$ with the arc $F_{\mathfrak{p}}$. 
9.1.1. Proposition. (existence of a uniform sufficient jet order, [Z02a, Theorem 2.1]) For any $r \geq 1$ there exists $R$ such that if $\mathfrak{p}, \mathfrak{p}^{\prime} \in T_{r}$ and $\left(F_{\mathfrak{p}} . F_{\mathfrak{p}^{\prime}}\right) \geq R+1$, then $s_{\mathfrak{p}}(L / K)=s_{\mathfrak{p}^{\prime}}(L / K)$. Let $\mathrm{su}_{1, r}(L / K)$ be the minimal such $R$. Then there exists $N \geq 1$ such that $\operatorname{su}_{1, r}(L / K)<N r$ for any $r$.

9.1.2. Remark. There was a mistake in the proof of "sufficient jet order conjecture" in [Z02b]. The correct part of this preprint on the bounded growth of curve singularity invariants along certain tame and wild morphisms of surfaces was published later as [Z06].

Next, introduce Zariski topology in all $T_{r, n}$ as in Subsection 8.3. Then the following statements hold.

9.1.3. Proposition. (semi-continuity of a break, [Z02a, Theorems 2.2-2.4]) 1. Let $n \geq \operatorname{su}_{1, r}(L / K)$. Denote by $J_{n}(\mathfrak{p})$ the $n$-jet of the arc $F_{\mathfrak{p}}$. Then for any $s \geq 0$ the set

$$
\left\{J_{n}(\mathfrak{p}) \mid \mathfrak{p} \in T_{r} ; s_{\mathfrak{p}}(L / K) \leq s\right\}
$$

is a closed subset in $T_{r, n}$.

2. The supremum

$$
s_{r}(L / K)=\sup \left\{s_{\mathfrak{p}}(L / K) \mid \mathfrak{p} \in T_{r}\right\}
$$

is finite.

3. Assume in addition that $A$ is a G-ring. Then the sequence $\left(s_{r}(L / K) / r\right)_{r}$ is convergent.

9.2. Extensions of prime degree. The paper $[\mathrm{Fa}]$ is devoted to morphisms $h$ : $A \rightarrow B$ of Subsection 8.3 with properties (i), (ii) and (iii) without assumption that $B$ is a Galois algebra over $A$.

Let $T_{r}, T_{r, R}, C, n(C), C_{i}^{\prime}$ have the same meaning as in Subsection 8.3. Under the assumption $n(C)=1$, denote by $s_{C}$ the only ramification break of $k\left(C_{1}^{\prime}\right) / k(C)$ as defined at the very end of $\S 1$. Then we have ([Fa, Theorem 4]):

9.2.1. Proposition. (existence of a uniform sufficient jet order) For any $r \geq 1$ there exists $R$ such that if $C, \tilde{C} \in T_{r}$ and $(C . \tilde{C}) \geq R+1$, then $s_{C}=s_{\tilde{C}}$. Let $\operatorname{su}_{1, r}(h)$ be the minimal such $R$. Then there exists $N \geq 1$ such that $\mathrm{su}_{1, r}(h)<N r$ for any $r$.

Next, I. Faizov proved the following semi-continuity statement ([Fa, Theorems 5 and 6]).

9.2.2. Proposition. (semi-continuity of a break) 1. Let $n \geq \mathrm{su}_{1, r}(h)$. Then for any rational $s \geq 0$ the set

$$
\left\{J_{n}(C) \mid C \in T_{r} ; s_{C} \leq s\right\}
$$

is a closed subset in $T_{r, n}$.

2. The supremum

$$
s_{r}(h)=\sup \left\{s_{C} \mid C \in T_{r}\right\}
$$

is finite.

The proofs are based on careful work with Hamburger-Noether algorithm for curve $C_{1}$ yielding an explicit form of a uinformizing element of $k(C)$. 
9.3. Relation to singularity invariants. In the context of Subsection 8.3, we considered regular arcs on $\operatorname{Spec} A$; however, the $\operatorname{arcs} C_{i}^{\prime}$ on $\operatorname{Spec} B$ are in general singular, and the complexity of singularity can reflect the ramification data of the morphism $h$; this phenomenon was first observed in [Z06]. In [CZ] we relate the semi-continuity property of ramification invariants with the semi-continuity of $\delta$ invariant in families of singular arcs.

Let $A, B$ be complete 2-dimensional regular local rings with algebraically closed coefficient subfield $k$. A finite $k$-homomorphism $h: A \rightarrow B$ will be called unmixed if $h\left(\mathfrak{m}_{A}\right) \subset \mathfrak{m}_{B}$ and $h\left(\mathfrak{m}_{A}\right) \not \subset \mathfrak{m}_{B}^{2}$. In particular, a homomorphism with properties (i) and (ii) is unmixed if in its definition either $e_{x}=1$ or $e_{y}=1$.

A decomposable homomorphism is by definition a composition of several unmixed homomorphisms.

The following statement is proved in [CZ].

9.3.1. Proposition. Let $h: A \rightarrow B$ be a decomposable homomorphism of degree $m$, and $\mathcal{B}$ its branch divisor in $\operatorname{Spec} A$. Let $C$ be a reduced curve on $\operatorname{Spec} A$ having no common components with $\mathcal{B} ; C^{\prime}=h^{*} C$. Let $C_{1}^{\prime}, \ldots, C_{r}^{\prime}$ be all components of $C^{\prime} ; C_{i}=h_{*} C_{i}^{\prime}, i=1, \ldots, r ; d_{i}$ the order of different in the extension of discrete valuation fields $k\left(C_{i}^{\prime}\right) / k\left(C_{i}\right)$. Then we have

$$
2 \delta\left(C^{\prime}\right)-2 m \delta(C)=(C . \mathcal{B})-\sum_{i=1}^{r} d_{i}
$$

This immediately implies

9.3.2. Corollary. Let $h: A \rightarrow B$ be a decomposable homomorphism, and $\mathcal{B}$ its branch divisor in $\operatorname{Spec} A$. Let $C$ be a regular curve on $\operatorname{Spec} A$ which is not a component of $\mathcal{B} ; C_{1}^{\prime}, \ldots, C_{r}^{\prime}$ all components of $C^{\prime}=h^{*} C, i=1, \ldots, l ; d_{i}$ the order of different in the extension of discrete valuation fields $k\left(C_{i}^{\prime}\right) / k(C)$. Then

1. $\sum_{i=1}^{r} d_{i} \leq(C \cdot \mathcal{B})$

2. $\delta\left(C^{\prime}\right) \leq \frac{1}{2}(C \cdot \mathcal{B})$.

Consider a decomposable homomorphism $h: A \rightarrow B$ and assume that the branch divisor of $h$ is of the form $\mathcal{B}=b D_{0}$, where $D_{0}$ is a regular reduced irreducible curve on $\operatorname{Spec} A$ and $b$ is a positive integer. (It is always so when $h$ has properties (i)-(iii) from Subsection 8.2.)

9.3.3. Lemma. Let $\Delta$ be a positive integer. Let $A$ be a complete 2-dimensional regular local ring having a coefficient subfield. Consider two curves $C, \tilde{C}$ on $\operatorname{Spec} A$ such that $\delta(C) \leq \Delta$, and $C, \tilde{C}$ have the same $2 \Delta$-jet. Let $C_{1}, \ldots, C_{r}$ be all irreducible components of $C$. Then $\tilde{C}$ also has $r$ irreducible components $\tilde{C}_{1}, \ldots, \tilde{C}_{r}$ with $\delta\left(\tilde{C}_{i}\right)=\delta\left(C_{i}\right)$ and $\left(\tilde{C}_{i} \cdot \tilde{C}_{j}\right)=\left(C_{i} . C_{j}\right)$ for all $i, j$.

9.3.4. Question. Is it possible to estimate Milnor and Tjurina numbers $\mu(C)$ or $\tau(C)$ in terms of $\delta(C)$ ? Maybe, one could apply formulas for $\mu(C)$ from [BGM], [MHW]. If yes, this would enable us to estimate finite determinacy of $C$.

Next, let $T_{r, R}, n(C), C_{i}^{\prime}$ have the same meaning as in Subsection 8.3.

9.3.5. Proposition. Let $C$ be a regular curve on $\operatorname{Spec} A$ with $\left(C . D_{0}\right)=r<\infty$. Then, for the curve $h^{*} C$, the number of components, their $\delta$-invariants and intersection numbers depend only on the jet of $C$ in $T_{r, b r}$. 
Proof. Let $C$ and $\tilde{C}$ have the same $b r$-jet. Then obviously $h^{*} C$ and $h^{*} \tilde{C}$ also have the same $b r$-jet. In view of Corollary 9.3.2, $\delta\left(h^{*} C\right) \leq b r / 2$. It remains to apply Lemma 9.3.3 with $\Delta=[b r / 2]$.

9.3.6. Corollary. For $C$ as in the above proposition, let $d_{i}$ be the order of different in the extension of discrete valuation fields $k\left(C_{i}^{\prime}\right) / k(C), i=1, \ldots, n(C)$. Then $\sum_{i=1}^{r} d_{i}$ depends only on the br-jet of $C$.

Proof. It follows from Proposition 9.3.5 and formula (16).

Let us make the following Assumption $S_{\delta}$ on the semi-continuity of the $\delta$ invariant.

Let $A$ be a complete 2-dimensional regular local ring with algebraically closed coefficient subfield $k$, and let $U$ be an open subset of $\mathbb{A}_{k}^{N}$ for some positive integer $N$. Let $f \in A\left[X_{1}, \ldots, X_{N}\right]$ be such that for any closed point $\left(a_{1}, \ldots, a_{N}\right) \in U$ the curve $C\left(a_{1}, \ldots, a_{N}\right)=\operatorname{Spec} A /\left(f, X_{1}-a_{1}, \ldots, X_{N}-a_{N}\right)$ is reduced. Assume that there exists a positive integer $\Delta$ such that $\delta\left(C\left(a_{1}, \ldots, a_{N}\right)\right) \leq \Delta$ for all $\left(a_{1}, \ldots, a_{N}\right) \in U$. Then $\delta\left(C\left(a_{1}, \ldots, a_{N}\right)\right)$ is an upper semi-continuous function on $U$.

9.3.7. Proposition. If Assumption $S_{\delta}$ is satisfied, then for any $r \geq 1, \delta\left(h^{*} C\right)$ determines an upper semi-continuous function on $T_{r, b r}$.

Proof. It follows immediately from Corollary 9.3.2.

9.3.8. Question. Is it true that $n(C)$ (the number of components of $h^{*} C$ ) determines a lower semi-continuous function on $T_{r, b r}$ ? What can be said about the generic value of $n(C)$ ?

9.3.9. Corollary. For a regular curve $C$ on $\operatorname{Spec} A$ with $\left(C . D_{0}\right)=r$, let $C_{1}^{\prime}, \ldots, C_{n}^{\prime}$ be all components of $h^{*} C, n=n(C)$, and $d_{i}$ the order of different in the extension of discrete valuation fields $k\left(C_{i}^{\prime}\right) / k(C)$. Then $\sum_{i=1}^{n} d_{i}$ determines a lower semicontinuous function on $T_{r, b r}$, if the Assumption $S_{\delta}$ is satisfied.

Proof. It follows immediately from Prop.9.3.7 and 9.3.1, since $(C . \mathcal{B})=b r$.

9.3.10. Question. We suggest to say that a lower semi-continuous integer-valued function $h$ on a variety $S$ is purely lower semi-continuous if for every $N$ each component of the closed subset

$$
S_{N}=\{P \in S \mid h(P)<N\}
$$

has codimension $\leq 1$ in the respective component of $S_{N+1}$.

Is it true that $\sum_{i=1}^{n} d_{i}$ determines a purely lower semi-continuous function on $T_{r, b r}$ ? Equivalently, is $\delta\left(\beta_{r}, \ldots, \beta_{p r}\right)$ purely upper semi-continuous on $T_{r, b r}$ ? (Pure upper semi-continuity is defined similarly.)

This is related to Deligne's conjecture that the loci of exceptional values of ramification invariants are always hypersurfaces.

\section{Algebraic-geometric Consequence of AbBes-Saito filtration}

The theory of Abbes and Saito filtration has deep applications in algebraic geometry including Grothendieck-Ogg-Shafarevich type formulas for Euler characterstic of étale sheaves. A survey of geometric applications is also given in the ICM talk of Saito [Sa10]. Here we prefer to discuss the global version of all three objects in Section 6 at the same time to emphasize their similarities. 
10.1. Setup. Let $k$ be a field. Let $X$ a smooth variety over $k$ and let $D=\cup_{i=1}^{r} D_{i}$ be a divisor on $X$ with strict simple normal crossings, where $D_{i}$ are irreducible components. Let $U=X \backslash D$ be the complement. Suppose that we are in one of the following globalizations of the three situations considered in Section 6:

(a) $\mathcal{F}$ is a lisse $\mathbb{Q}_{l}$-sheaf on $U$, where $l$ is a prime number different from char $k$;

(b) $\mathcal{F}$ is an $F$-isocrystal on $U$ overconvergent along $D$, while char $k=p>0$;

(c) $\mathcal{F}$ is a locally free coherent sheaf on $U$ with an integrable connection, while char $k=0$.

At the generic point $\eta_{i}$ of an irreducible component $D_{i}$ of the divisor $D$, one can talk about

(a) the Swan conductor $\operatorname{Sw}\left(\mathcal{F} ; D_{i}\right)$, obtained by considering the representation $G_{k(X)^{\eta_{i}}} \rightarrow \pi_{1}(U) \rightarrow G L\left(V_{\mathcal{F}}\right)$, where the latter homomorphism is the representation associated to the lisse sheaf $\mathcal{F}$; or

(b) the (differential) Swan conductor $\operatorname{Sw}\left(\mathcal{F} ; D_{i}\right)$, obtained by passing to the generic point in the sense of Subsection 6.2 ; or

(c) irregularity $\operatorname{Irr}\left(\mathcal{F} ; D_{i}\right)$ in the sense of Subsection 6.3 by base changing to the completion at $\eta_{i}$; we rename it the Swan conductor $\operatorname{Sw}\left(\mathcal{F} ; D_{i}\right)$.

We list all these three cases together because most of results on ramification theory hold in similar fashion.

10.2. Results of variation. We explain the main result of [KeX, Ke11a, Ke10b] on variation of Swan conductors by an example. We take $X=\mathbb{A}^{2}=\operatorname{Speck}[x, y]$, $D_{0}=V(y)$ and $D_{1}=V(x)$. Let $\mathcal{F}$ be an object over $U=X \backslash\left(D_{0} \cup D_{1}\right)$ as above. We can consider the Swan conductors $\operatorname{Sw}\left(\mathcal{F} ; D_{0}\right)$ and $\operatorname{Sw}\left(\mathcal{F} ; D_{1}\right)$.

We may blowup $X$ at the origin $P=D_{0} \cap D_{1}$ to get $X^{\prime}=\mathrm{Bl}_{P} X$; let $D_{1 / 2}$ denote the exceptional divisor. Since $\mathcal{F}$ is defined on $U$, we can talk about the Swan conductor $\operatorname{Sw}\left(\mathcal{F} ; D_{1 / 2}\right)$. Carrying on this idea, we can continue to blowup $X^{\prime}$ along the intersections of $D_{1 / 2}$ with the proper transfer of $D_{0}$ and $D_{1}$. We use $D_{1 / 3}$ and $D_{2 / 3}$ to denote the two exceptional divisors for this blowup. Similarly, we can talk about the Swan conductors $\operatorname{Sw}\left(\mathcal{F} ; D_{1 / 3}\right)$ and $\operatorname{Sw}\left(\mathcal{F} ; D_{2 / 3}\right)$. We can iterate this process to blowup intersections of the divisors and then consider the Swan conductors along all the exceptional divisors. We label the exceptional divisors as follows: for each coprime pair $(m, n) \in \mathbb{N}$, there is exactly one such exceptional divisor $D_{n / m+n}$ such that, for the valuation $v$ corresponding to $D_{n / m+n}$, we have $v(x)=n$ and $v(y)=m$. Along this divisor, we have a $\operatorname{Swan}$ conductor $\operatorname{Sw}\left(\mathcal{F} ; D_{n / m+n}\right)$.

10.2.1. Proposition. The function

$$
\frac{n}{n+m} \longmapsto \frac{1}{n+m} \operatorname{Sw}\left(\mathcal{F} ; D_{n / m+n}\right)
$$

extends by continuity to a convex piecewise linear function on $[0,1]$ with integral slopes.

This proposition is a special case of the result for higher dimensional $X$ and for an intersection point of simple normal crossing divisors, as proved in [KeX, Ke11a, Ke10b]. (The essential part of the proof is in $[\mathrm{KeX}]$. But the statements appear in [Ke11a] for cases (a) and (b) and in [Ke10b] for case (c).) Moreover, the slopes of the piecewise linear function are related to the refined Swan conductor homomorphism, as proved in Subsection 5.4. (See [X12b] for details.) 
10.2.2. Remark. We point out a caveat: there is no analogous result of Proposition 10.2.1 for Artin conductors. One of the explanations could be: blowing up is log-smooth but not smooth. So a log version conductor is expected to work much better.

10.3. Approach to ramification theory using cutting-by-curves. It would be interesting to point out the conjectural relation between the generic ramification using Abbes-Saito filtration (discussed above) and the ramification data from cutting-by-curves (as discussed in detail in Section 9).

The Abbes-Saito Swan conductor is defined by looking at the generic points of the divisors, as explained in Subsection 10.1. We now explain the "cut-by-curve" Swan conductors. Let $D_{i}$ be an irreducible divisor of $X$, then one can define a new Swan conductor by taking

$$
\operatorname{Sw}_{\text {curve }}\left(\mathcal{F} ; D_{i}\right):=\sup _{C}\left(\frac{\operatorname{Sw}\left(\left.\mathcal{F}\right|_{C} ; C \cap D_{i}\right)}{\left(C . D_{i}\right)}\right)
$$

where $\left(C . D_{i}\right)$ is the intersection number of $C$ with $D_{i}$ and the supremum is taken over all curves $C$ that intersects with $D_{i}$ (not necessarily transversely). A suggestion to study $\mathrm{Sw}_{\text {curve }}$ appeared (in 2-dimensional case) in [Z02b, Remark 2.5.3]; a computation in the Artin-Schreier case was done in [Z02a] (see above Prop. 9.1.3).

The natural question to ask is whether $\operatorname{Sw}_{\text {curve }}\left(\mathcal{F} ; D_{i}\right)$ is the same as $\operatorname{Sw}\left(\mathcal{F} ; D_{i}\right)$ using the Abbes-Saito ramification filtration. We do not discuss this comparison in detail, but refer to the forthcoming thesis [Ba] of I. Barrientos, which generalizes an idea of Deligne-Esnault-Kerz [EK]. We nevertheless will emphasize that using curves that are not transversal to the divisor is necessary in this theory, as shown in the following example.

10.3.1. Example. Let $X=\mathbb{A}^{2}$ be the $x y$-plane over a field $k$ of characteristic $p$ and let $D$ be the divisor $Z(y)$. Consider the Artin-Schreier sheaf $\mathcal{F}$ over $U=X-D$ given by the equation $z^{p}-z=x / y^{p}$, that is the lisse sheaf associated to a nontrivial representation of the Galois group $\mathbb{Z} / p \mathbb{Z}$ given by this equation.

Using Example 5.3.2, we see that $\operatorname{Sw}(\mathcal{F} ; D)=p$. (Note that $x$ is not a $p$ th power in the residue field $k(x)$.) However, when restricted to each line $C_{a}: x=a$ for $a \in k^{\text {alg }}$, the Artin-Schreier equation becomes $z^{p}-z=a / y^{p}$ which is the same as $z^{\prime p}-z^{\prime}=a^{1 / p} / y$ for $z^{\prime}=z-a^{1 / p} / y$. So $\operatorname{Sw}\left(\left.\mathcal{F}\right|_{C_{a}} ; D \cap C_{a}\right)=1$. In other words, the generic Swan conductor (using Abbes-Saito's filtraiton) does not equal to the Swan conductor restricted to any such curve $C_{a}$.

If instead we consider the curve $C_{a, m}: y=(x-a)^{m}$ for $a \in k^{\text {alg }}$ and $m \gg 0$, the Artin-Schreier equation becomes $z^{p}-z=x /(x-a)^{p m}$. It is easy to compute that $\operatorname{Sw}\left(\left.\mathcal{F}\right|_{C_{a, m}} ; D \cap C_{a, m}\right)=p m-1$. So $\lim \sup _{m} \frac{\operatorname{Sw}\left(\left.\mathcal{F}\right|_{C_{a, m}} ; D \cap C_{a, m}\right)}{\left(D \cdot C_{a, m}\right)}=p$.

We also point out that when $m=1$, the curve $y=x-a$ is still transversal to $D$, but $\operatorname{Sw}\left(\left.\mathcal{F}\right|_{C_{a, 1}} ; D \cap C_{a, 1}\right)=p-1$, which is different from $\operatorname{Sw}\left(\left.\mathcal{F}\right|_{C_{a}} ; D \cap C_{a}\right)=1$; thus restricting to different transversal curves may give different Swan conductors. The largest Swan conductor obtained by restricting to transversal curves is $p-1$, which is still smaller than the "correct answer" $p$, as seen at the "generic point". This is why we need to consider curves non-transversal to the divisor. 
10.3.2. Question. Using the result on variation of Abbes-Saito Swan conductor (Prop. 10.2.1) and the information of refined Swan conductors, can we say something along the line of semi-continuity type statement proposed by Deligne [De76] (and proved by $[\mathrm{La}]$ in case of absence of ferocious ramification)?

10.4. Towards a generalized Grothendieck-Ogg-Shafarevich formula. One of the goals of Abbes and Saito's project is to generalize the Euler characteristic formula for $l$-adic sheaves (Grothendieck-Ogg-Shafarevich type formula). For us, this applies to all three cases we discussed above. We will only discuss formulas that involve only the Swan conductors discussed in Section 6 .

The formula is known when $X$ is a curve. Case (a) is discussed in Subsection 3.11. Case (b) is due to Christol, Crew, Matsuda, Mebkhout, and Tsuzuki; a complete reference with a proof is given in [Ke06, Theorem 4.3.1]. Case (c) is due to Deligne and Gabber; one can find a proof in [Katz, Theorem 2.9.9].

For $X$ general, the $l$-adic conjecture is described in detail in [AS11, Sa10] and proved in a special case in [Sa09]. It is proved in the case of vector bundles with flat connections under probably a necessary condition (see $[\mathrm{X} 12+]$ ). We sketch the key points involved.

First, it appears to be impossible to obtain an unconditional formula that resembles the classical Grothendieck-Ogg-Shafarevich formula (9) and involves only the geometry of $X$ and the Swan conductors (or more generally the set of highest ramification breaks of constituents) discussed in Section 6. This is because the ramification data at the generic points of the divisors do not determine the ramification at closed points. One has to impose a cleanness ${ }^{6}$ condition on the object $\mathcal{F}$, which roughly says that the ramification at all closed points on $D$ is determined by the ramification data at generic points of $D$. The cleanness condition is discussed in [AS11] for $l$-adic sheaves, but also note the subtlety of different versions of cleanness, as discussed in $[\mathrm{X} 12+]$ for case $(c)$.

10.4.1. Question. Does there always exist a birational proper morphism $f:\left(X^{\prime}, D^{\prime}\right) \rightarrow$ $(X, D)$ such that $f^{*} \mathcal{F}$ satisfies the aforementioned cleanness condition? In case $(\mathrm{c})$, this question is known as the Sabbah's Conjecture, proved by Kedlaya [Ke10b, Ke11b] and T. Mochizuki [Mo-T] independently.

Second, assuming the cleanness condition, the ramification data does not only give the Euler characteristic, but it is also expected to determine log-characteristic cycle of $\mathcal{F}$ (as a cycle in the log-cotangent space of $X$ ). The definition of logcharacteristic cycles is in fact a big mystery, which we will discuss in the next paragraph; for now, we just explain its conjectural properties assuming its existence. Unlike in the usual (nonlog) characteristic cycle for algebraic $D$-modules (for definition of characteristic cycles, see, e. g., [HTT]), where all irreducible components are conormal bundles of some closed subvariety of $X$, the log-characteristic cycles are supposed to "point to various directions" in the cotangent space, determined by the refined Swan conductors. This is related to the fact that the Poisson structure on the log-cotangent space is degenerate. However, the Euler characteristic does not depend on the "directions".

In standard theory of algebraic $D$-modules and overconvergent $F$-isocrystals, the intersection number of the characteristic cycle with the zero section of the

\footnotetext{
${ }^{6}$ Abbes and Saito used the word "cleanliness"; but we feel "cleanness" is a more appropriate word here.
} 
cotangent space gives the Euler characteristic of $\mathcal{F}$; this formula is known as the Kashiwara-Dubson formula. See [HTT] for the algebraic $D$-module case and [Be] for the overconvergent $F$-isocrystal case. One may hope to use a log-version of such a formula to prove the generalized Grothendieck-Ogg-Shafarevich formula by computing explicitly the log-characteristic cycles, at least in the case of $F$-isocrystals and algebraic $D$-modules. However, the technical subtlety here is exactly that, unlike in the standard nonlog case, the log-characteristic cycles are not known to be well-defined for $F$-isocrystals. Even for vector bundles with flat connections, the theory of log-characteristic cycles is quite different from the classical theory. The major difficulty is the lack of log-holonomicity! See $[\mathrm{X} 12+]$ for more discussion.

10.4.2. Remark. A very important application for an appropriate definition of log-characteristic cycles for overconvergent $F$-isocrystals would be the following. Kedlaya developed a trick in [Ke11a, Section 5] that can "transfer" the ramification data of a lisse $l$-adic sheaf to a (virtual) overconvergent $F$-isocrystal. Then we would get a natural definition of log-characteristic cycles for lisse $l$-adic sheaves for free. To our knowledge, a general construction of (log-)characteristic is not known for lisse $l$-adic sheaves. (Under the cleanness condition, Abbes and Saito [AS11] gave a definition using the refined Swan conductors, but it is unclear how to relate this, for example, to the analog of the supersingular supports.)

\section{Miscellaneous QUestions}

11.1. Ramification numbers and structure of Galois groups. There exists a number of results relating the structure of Galois groups with the possible values of ramification invariants. Hasse-Arf theorem gives an example; another example is the following Hyodo inequality ([Hy, Lemma (4-1)] or, without class field theory, $[\mathrm{Z} 95, \S 1])$.

11.1.1. Proposition. Let $M / K$ be a cyclic extension $p^{2}, L$ the intermediate subfield. Then

$$
d_{K}(M / L) \geq \min \left(\left(p-1+p^{-1}\right) d_{K}(L / K), e_{K}-p^{-1} e_{K}+p^{-1} d_{K}(L / K)\right) .
$$

11.1.2. Question. Given a complete discrete valuation field $K$, a word $T=T_{1} \ldots T_{n}$ in the alphabet $\{W, F\}$ and an $n$-tuple of integers $\left(i_{1}, \ldots, i_{n}\right)$, does there exist a cyclic extension $L / K$ with genome $T$ and lower breaks $\left(i_{1}, \ldots, i_{n}\right)$ ?

The answer is known only in 2 cases.

(1) The classical case: we can give only the reference [Mik81] for the mixed characteristic case. For equal characteristic case, a related work is [Th].

(2) Ferocious extensions of 2-dimensional fields ([We]).

In general, we do not even know the answer to the following question.

11.1.3. Question. Given a complete discrete valuation field $K$ and a word $T=$ $T_{1} \ldots T_{n}$ in the alphabet $\{W, F\}$, does there exist a cyclic extension $L / K$ with genome $T$ ?

If char $K=p$, the answer is thought to be positive for any $T$; however, it cannot be so if char $K=0$. Indeed, according to [Kur], in this case any complete discrete valuation field belongs to one of two types; the fields of type I (resp. of type II) do not have arbitrarily big cyclic ferocious (resp. wild) extensions. It would be 
interesting to try to answer Question 11.1.3 in terms of refinement of Kurihara's classification by O. Ivanova [I12].

One more aspect of this topic is the following phenomenon in the mixed characteristic case: the assumption that the minimal ramification break of $L / K$ takes its almost maximal value, namely, $h \geq \frac{p e_{K}}{p-1}-1$, has strong implications for the whole ramification filtration; see [PVZ] for a number of results in this direction.

11.2. Small ramification numbers and embedding problem. In this subsection, we assume char $K=0$.

By a result of Miki [Mik74], if $L / K$ is a cyclic extension of degree $p$, it can be embedded into a cyclic extension of degree $p^{n}$ if and only if $L\left(\zeta_{p}\right)=K_{1}(x)$, where $x^{p} \in N_{K_{n} / K_{1}} K_{n}^{*}$, and $K_{n}$ denotes $K\left(\zeta_{p^{n}}\right)$. The following statement is an easy consequence $([\mathrm{VZ}, \S 2])$.

11.2.1. Proposition. Let $L / K$ is a cyclic extension of degree $p$ with $d_{K}(L / K)<$ $\frac{e_{K}}{p-1}$. Then $L / K$ can be embedded into a cyclic extension of degree $p^{2}$.

We are interested in generalization of this observation to any Galois groups.

11.2.2. Question. Let $f: G^{\prime} \rightarrow G$ be an epimorphism of finite groups. Does there exist an $\varepsilon_{f}>0$ such that, for any Galois extension $L / K$ of mixed characteristic complete discrete valuation fields with $\operatorname{Gal}(L / K) \simeq G$ and $d_{K}(L / K)<\varepsilon_{f} e_{K}$, the embedding problem $(L / K, f)$ has a solution?

11.3. Ramification and higher adèles. It would be interesting to understand what kind of ramification data are needed in adelic theory of arithmetic surfaces. For example, the non-wild part of the conductor of the curve appears in [Fe10, Subsection 3.4]; can we allow wild ramification here?

\section{REFERENCES}

[AM] A. Abbes, A. Mokrane, Sous-groupes canoniques et cycles évanescents p-adiques pour les variétés abéliennes, Publ. Math. Inst. Hautes Etudes Sci. 99 (2004), 117-162.

[AS02] A. Abbes, T. Saito, Ramification of local fields with imperfect residue fields. I, Amer. J. Math. 124 (2002), 879-920, arXiv:math/0010103.

[AS03] A. Abbes, T. Saito, Ramification of local fields with imperfect residue fields. II, Kazuya Kato's fiftieth birthday. Doc. Math. 2003, Extra Vol., 5-72 (electronic).

[AS09] A. Abbes, T. Saito, Analyse micro-locale l-adique en caractristique $p>0$ : Le cas d'un trait, Publication of the Research Institute for Mathematical Sciences 45 (2009), no. $1,25-74$.

[AS11] A. Abbes, T. Saito, Ramification and cleanliness, Tohoku Mathematical Journal (2) 63 (2011), no. 4, 775-853.

[Abr00] V. A. Abrashkin, On a local analogue of the Grothendieck conjecture, Internat. J. Math. 11, (2000), 133-175.

[Abr02] V. A. Abrashkin, Ramification theory for higher dimensional local fields, in book: Algebraic number theory and algebraic geometry, 1-16, Contemp. Math., 300, Amer. Math. Soc., Providence, RI, 2002.

[Abr03] V. A. Abrashkin, An analogue of Grothendieck's conjecture for two-dimensional local fields of finite characteristic, Tr. Mat. Inst. Steklova 241 (2003), 8-42; translation in Proc. of Steklov Institute 241 (2003), 2-34.

[Abr07] V. A. Abrashkin, An analogue of the field-of-norms functor and of the Grothendieck conjecture, J. Algebraic Geom. 16 (2007), no. 4, 671-730, arXiv:math/0503200.

[Abr10] V. A. Abrashkin, Modified proof of a local analogue of the Grothendieck conjecture, Journal Theorie des Nombres de Bordeaux 22 (2010), 1-50, arXiv:0907.3035.

[Ba] I. Barrientos, thesis. 
[Be] P. Berthelot, Introduction à la théorie arithmétique des D-modules, , Astérisque $\mathbf{2 7 9}$ (2002), 1-80.

[BCS] R. Boltje, G.-M. Cram, and V. P. Snaith, Conductors in the non-separable residue field case, in book: Algebraic K-theory and algebraic topology (Lake Louise, AB, 1991), 134, NATO Adv. Sci. Inst. Ser. C Math. Phys. Sci., 407, Kluwer Acad. Publ., Dordrecht, 1993.

[BGR] S. Bosch, U. Güntzer, and R. Remmert. Non-Archimedean analysis: a systematic approach to rigid analytic geometry, Grundlehren der Mathematischen Wissenschaften, vol. 261, Springer-Verlag, Berlin, 1984

[BGM] Y. Boubakri, G.-M. Greuel, T. Markwig, Invariants of hypersurface singularities in positive characteristic, Rev. Mat. Complut. 25 (2012), no. 1, 61-85, arXiv:1005.4503.

[Bo02] J. M. Borger, Kato's conductor and generic residual perfection, preprint (2002), arXiv: math/0112306.

[Bo04] J. M. Borger, Conductors and the moduli of residual perfection, Math. Ann. 329 (2004), 1-30, arXiv:math/0112305.

[Br] J.-L. Brylinski, Théorie du corps de classes de Kato et revêtements abéliens de surfaces, Ann. Inst. Fourier, Grenoble 33 (1983), 23-38.

[ChP] B. Chiarellotto and A. Pulita, Arithmetic and differential Swan conductors of rank one representations with finite local monodromy, Amer. J. Math. 131 (2009), no. 6, 17431794.

[CuP] S. D. Cutkosky, O. Piltant, Ramification of valuations, . Adv. Math. 183 (2004), no. $1,1-79$.

[CZ] A. Campillo, I. B. Zhukov, Curve singularities and ramification of surface morphisms, in preparation.

[De76] P. Deligne, Letter to L. Illusie of 28.11.76, unpublished.

[De84] P. Deligne, Les corps locaux de caractéristique $p$, limites de corps locaux de caractéristique 0, in book: Representations of reductive groups over a local field, 119-157, Travaux en Cours, Hermann, Paris, 1984.

[dS] B. de Smit, Ramification groups of local fields with imperfect residue class field, J. Number Theory 44 (1993), no. 3, 229-236.

[E] H. Epp, Eliminating wild ramification, Invent. Math. 19 (1973), 235-249.

[EK] H. Esnault, M. Kerz, A finiteness theorem for Galois representations of function fields over finite fields (after Deligne), , preprint (2012), arXiv:1208.0128.

[Fa] I. Faizov, Properties of ramification jump in extensions of degree $p$, submitted to Zap. Nauchn. Sem. POMI.

[Fe95a] I. B. Fesenko, Abelian local p-class field theory, Math. Ann. 301 (1995), no. 3, 561-586.

[Fe95b] I. B. Fesenko, Hasse-Arf property and abelian extensions, Math. Nachr. 174 (1995), $81-87$.

[Fe96] I. B. Fesenko, Abelian extensions of complete discrete valuation fields, in book: Number theory (Paris, 19931994), 47-74, London Math. Soc. Lecture Note Ser., 235, Cambridge Univ. Press, Cambridge, 1996.

[Fe01] I. B. Fesenko, Nonabelian local reciprocity maps, in book: Class field theoryits centenary and prospect (Tokyo, 1998), 63-78, Adv. Stud. Pure Math., 30, Math. Soc. Japan, Tokyo, 2001.

[Fe10] I. B. Fesenko, Analysis on arithmetic schemes. II, J. K-Theory 5 (2010), no. 3, 437-557.

[FV] I. B. Fesenko and S. V. Vostokov, Local fields and their extensions. A constructive approach, Second edition, AMS, Providence, RI, 2002.

[FW1] J.-M. Fontaine and J.-P. Wintenberger Extensions algébrique et corps des normes des extensions APF des corps locaux, C. R. Acad. Sci. Paris Sér. A-B 288 (1979), no. 8, A441-A444.

[FW2] J.-M. Fontaine and J.-P. Wintenberger, Le "corps des normes" de certaines extensions algébriques de corps locaux, C. R. Acad. Sci. Paris Sér. A-B 288 (1979), no. 6, A367A370.

[Ha12] S. Hattori, Ramification correspondence of finite flat group schemes over equal and mixed characteristic local fields, J. Number Theory 132 (2012), no. 10, 2084-2102.

[Ha12+] S. Hattori, On lower ramification subgroups and canonical subgroups, preprint (2012), arXiv:1208.5326. 
[HLF] Invitation to higher local fields (Münster, 1999), Geom. Topol. Monogr., 3, Geom. Topol. Publ., Coventry, 2000; www.maths.warwick.ac.uk/gt/gtmcontents3.html.

[Hi] T. Hiranouchi, Ramification of truncated discrete valuation rings: a survey, in book: Algebraic number theory and related topics 2008, 35-43, RIMS Kôkyûroku Bessatsu, B19, Res. Inst. Math. Sci. (RIMS), Kyoto, 2010.

[HT] T. Hiranouchi, Y. Taguchi, Extensions of truncated discrete valuation rings, Pure Appl. Math. Q. 4 (2008)), 1205-1214.

[HTT] R. Hotta, K. Takeuchi, and T. Tanisaki, D-modules, perverse sheaves, and representation theory, Translated from the 1995 Japanese edition by Takeuchi, Progress in Mathematics, vol. 236, Birkhäuser Boston, Inc., Boston, MA, 2008.

[Hy] O. Hyodo, Wild ramification in the imperfect residue field case, Adv. Stud. Pure Math. 12 (1987), 287-314.

[108] O. Yu. Ivanova, The rank of a topological $K$-group as a $\mathbb{Z}_{p}$-module, , Algebra i Analiz 20 (2008), no. 4, 87-117; translation in St. Petersburg Math. J. 20 (2009), no. 4, 569-591.

[I12] O. Yu. Ivanova, On relation between Kurihara classification and theory of elimination of ramification, Algebra i Analiz 24 (2012), 130-153.

[IS] K. I. Ikeda, E. Serbest, Ramification theory in non-abelian local class field theory, Acta Arith. 144 (2010), no. 4, 373-393.

[Ka87] K. Kato, Vanishing cycles, ramification of valuation and class field theory, Duke Math. J. 55 (1987), 629-659.

[Ka89] K. Kato, Swan conductors for characters of degree one in the imperfect residue field case, in book: Algebraic K-theory and algebraic number theory (Honolulu, HI, 1987), 101-131, Contemp. Math., vol. 83, Amer. Math. Soc., Providence, RI, 1989.

[Katz] N. M. Katz, Exponential sums and differential equations, Annals of Mathematics Studies, vol. 124. Princeton University Press, Princeton, NJ, 1990.

[Ka94] K. Kato, Class field theory, $\mathcal{D}$-modules, and ramification on higher-dimensional schemes. I., Amer. J. Math. 116 (1994), no. 4, 757-784.

[Ke05] K. S. Kedlaya, Local monodromy of p-adic differential equations: an overview, International Journal of Number Theory 1 (2005), no. 1, 109-154.

[Ke06] K. S. Kedlaya, Fourier transforms and p-adic "Weil II", Compositio Mathematica 142 (2006), no. 6, 1426-1450.

[Ke07] K. S. Kedlaya, Swan conductors for p-adic differential modules. I. A local construction, Algebra Number Theory 1 (2007), no. 3, 269-300.

[Ke10a] K. S. Kedlaya, p-adic Differential Equations, Cambridge Studies in Advanced Mathematics, vol. 125, Cambridge Univ. Press, 2010.

[Ke10b] K. S. Kedlaya, Good formal structures for flat meromorphic connections, I: Surfaces, Duke Mathematical Journal 154 (2010), no. 2, 343-418.

[Ke11a] K. S. Kedlaya, Swan conductors for p-adic differential modules, II: Global variation, Journal de l'Institut de Mathematiques de Jussieu 10 (2011), no. 1, 191-224.

[Ke11b] K. S. Kedlaya, Good formal structures for flat meromorphic connections, II: Excellent schemes, Journal of the American Mathematical Society 24 (2011), no. 1, 183-229.

[KeX] K. S. Kedlaya, L. Xiao, Differential modules on p-adic polyannuli, J. Inst. Math. Jussieu 9 (2010), no. 1, 155-201, arXiv:0804.1495.

[Kö] B. Köck, Computing the equivariant Euler characteristic of Zariski and étale sheaves on curves, Homology, Homotopy Appl. 7 (2005), no. 3, 83-98.

[Kuhl] F.-V. Kuhlmann, A correction to: "Elimination of wild ramification" by H. P. Epp, Invent. Math. 153 (2003), no. 3, 679-681.

[Kur] M. Kurihara, On two types of complete discrete valuation fields, Compositio Math. 63 (1987), 237-257.

[KZ] M. V. Koroteev and I. B. Zhukov, Elimination of wild ramification, Algebra i Analiz 11 (1999), 153-177; translation in St. Petersburg Math. J. 11 (2000), 1063-1083.

[La] G. Laumon, Semi-continuité du conducteur de Swan (d'après P. Deligne), Astérisque 83 (1981), 173-219.

[Lo] V. G. Lomadze, On the ramification theory of two-dimensional local fields, Mat. Sb. (N.S.) 109(151) (1979), no. 3, 378-394, 478; translation in Math. USSR Sbornik 37 (1980), 349-365.

[Ma] S. Matsuda, On the Swan conductor in positive characteristic, Amer. J. Math., 119 (1997), no. 4, 705-739. 
[MHW] A. Melle-Hernández; C. T. C. Wall, Pencils of curves on smooth surfaces, Proc. London Math. Soc. (3) 83 (2001), 257-278.

[Mik74] H. Miki, On $\mathbb{Z}_{p}$-extensions of complete p-adic power series fields and function fields, J. Fac. Sci. Univ. Tokyo, Sect 1A 21 (1974), 377-393.

[Mik81] H. Miki, On the ramification numbers of cyclic p-extensions over local fields, J. Reine Angew. Math. 328 (1981), 99-115.

[Mil] J. Milne, Étale cohomology, Princeton University Press, Princeton, 1980.

[Mo-S] Sh. Mochizuki, A version of the Grothendieck conjecture for p-adic local fields, Int. J. Math. 8 (1997), 499-506.

[Mo-T] T. Mochizuki, Wild harmonic bundles and wild pure twistor D-modules., Astérisque 340 (2011), $\mathrm{x}+607$.

[P] K. N. Ponomaryov, Solvable elimination of ramification in extensions of discretely valued fields, Algebra i Logika 37 (1998), no. 1, 63-87, 123; translation in Algebra and Logic 37 (1998), no. 1, 35-47.

[PVZ] G. K. Pak, S. V. Vostokov, I. B. Zhukov, Extensions with almost maximal depth of ramification, Zap. Nauchn. Sem. S.-Peterburg. Otdel. Mat. Inst. Steklov. (POMI) 265 (1999), 77-109; translation in J. Math. Sci. (New York) 112 (2002), no. 3, 4285-4302.

[Sa09] T. Saito, Wild ramification and the characteristic cycle of an l-adic sheaf, Journal de l'Institut de Mathematiques de Jussieu 8 (2009), 769-829, arXiv:0705.2799.

[Sa10] T. Saito, Wild ramification of schemes and sheaves, in book: Proceedings of the International Congress of Mathematicians (ICM 2010), Hyderabad, India, August 1927, 2010. Vol. II: Invited lectures, , 335-356, Hackensack, NJ: World Scientific; New Delhi: Hindustan Book Agency, 2011.

[Sa12] T. Saito, Ramification of local fields with imperfect residue fields. III, Math. Ann 352 (2012), 567-580, arXiv: 1005.2824.

[Sch] A. J. Scholl, Higher fields of norms and $(\phi, \Gamma)$-modules, Doc. Math. 2006, Extra Vol., 685-709 (electronic).

[Se68] J.-P. Serre, Corps Locaux, 2nd ed., Hermann, Paris, 1968.

[Se77] J.-P. Serre, Linear representations of finite groups, Graduate Texts in Mathematics, vol. 42, Springer-Verlag, New York-Heidelberg, 1977.

[Sn] V. P. Snaith, Explicit Brauer Induction, Cambridge Unicersity Press, 1994.

[Sp99] L. Spriano, Well and fiercely ramified extensions of complete discrete valuation fields, with applications to the Kato conductor, Thèse à 1 Université Bordeaux I, 1999.

[Sp00] L. Spriano, Well ramified extensions of complete discrete valuation fields with applications to the Kato conductor, Canad. J. Math. 52 (2000), 1269-1309.

[Th] L. Thomas, Ramification groups in Artin-Schreier-Witt extensions, J. Théor. Nombres Bordeaux 17 (2005), no. 2, 689-720.

[Ti] Y. Tian, Canonical subgroups of Barsotti-Tate groups, Ann. of Math. (2) 172 (2010), no. 2, 955-988.

[VZ] S. V. Vostokov, I. B. Zhukov, Some approaches to the construction of abelian extensions for $\mathfrak{p}$-adic fields, Proceedings of the St. Petersburg Mathematical Society, vol. III, 157174; translation in Amer. Math. Soc. Transl. Ser. 2, 166, Amer. Math. Soc., Providence, RI, 1995.

[We] S. Wewers, Fiercely ramified cyclic extensions of p-adic fields with imperfect residue field, preprint (2011), arXiv:1104.3785.

[Wh] W. A. Whitney Functorial Cohen Rings, Ph.D. thesis, University of California, Berkeley, 2002.

[X05] L. Xiao, Nonarchimedean differential modules and ramification theory, Ph.D. thesis, Massachusetts Institute of Technology, ProQuest LLC, Ann Arbor, MI, 2009.

[X10] L. Xiao, On Ramification Filtrations and p-adic Differential Equations, I: equal characteristic case, Algebra Number Theory 4 (2010), no. 8, 969-1027, arXiv:0801.4962.

[X12a] L. Xiao, On Ramification Filtrations and p-adic Differential Equations, II: mixed characteristic case, Compos. Math. 148 (2012), no. 2, 415-463, arXiv:0811.3792.

[X12b] L. Xiao, On refined ramification filtrations in the equal characteristic case, to appear in Algebra and Number Theory, arXiv:0911.1802.

$[\mathrm{X} 12+]$ L. Xiao, Cleanness and log-characteristic cycles, I: vector bundles with flat connections, preprint (2011), arXiv:1104.1224. 
[Z95] I. B. Zhukov, Structure theorems for complete fields, Proceedings of the St. Petersburg Mathematical Society, Vol. III, 175-192; translation in Amer. Math. Soc. Transl. Ser. 2, 166, Amer. Math. Soc., Providence, RI, 1995.

[Z97] I. B. Zhukov, Milnor and topological K-groups of higher-dimensional complete fields, Algebra i Analiz 9 (1997), 98-147; translation in St. Petersburg Math. J. 9 (1998), no. 1, 69-105.

[Z02a] I. B. Zhukov, Ramification of surfaces: Artin-Schreier extensions, in book: Algebraic number theory and algebraic geometry, , 211-220, Contemp. Math., 300, Amer. Math. Soc., Providence, RI, 2002.

[Z02b] I. B. Zhukov, Ramification of surfaces: sufficient jet order for wild jumps, preprint (2002), arXiv:math/0201071.

[Z03] I. B. Zhukov, On ramification theory in the case of an imperfect residue field, Mat. Sb. 194 (2003), 3-30; translation in Sb. Math. 194 (2003), no. 11-12, 1747-1774.

[Z06] I. B. Zhukov, Singularities of arcs and cyclic coverings of surfaces, Proceedings of the St. Petersburg Mathematical Society. Vol. XI, 49-66; translation in Amer. Math. Soc. Transl. Ser. 2, 218, Amer. Math. Soc., Providence, RI, 2006.

[Z10] I. B. Zhukov, Semiglobal models of extensions of two-dimensional local fields, Vestnik St. Petersburg Univ. Math. 43 (2010), no. 1, 33-38.

E-mail address: lxiao@math.uchicago.edu, zhukov@math.spbu.ru 